\title{
Seasonal and clock angle control of the location of flux transfer event signatures at the magnetopause
}

\author{
R. C. Fear, ${ }^{1}$ M. Palmroth, ${ }^{2}$ and S. E. Milan ${ }^{1}$ \\ Received 5 October 2011; revised 13 January 2012; accepted 3 February 2012; published 4 April 2012.
}

[1] Most models of flux transfer event (FTE) formation produce pairs of structures which in general move away from the subsolar region and give rise to signatures which can be observed in both the Northern and Southern Hemispheres. The multiple reconnection line (X line) model is unusual as a reconnection-based model that is capable of producing a single flux rope if only two X lines are present. Raeder (2006) reported the results of an MHD simulation where he studied the effect of the Earth's dipole tilt on reconnection at the dayside magnetopause for a southward IMF orientation; in his simulations, flux ropes were formed by the sequential formation of $\mathrm{X}$ lines, and when the dipole tilt was set to a value representative of solstice the flux ropes moved preferentially toward the winter hemisphere. Some observational evidence has previously been presented for a bias toward FTE signatures being observed in the winter hemisphere; in this paper, we present further observational evidence for this phenomenon, using an independently derived data set.

Once the seasonal bias is taken into account, we find that the IMF clock angle controls the location of FTE signatures. We also find that the effective dipole tilt (combining the geomagnetic dipole tilt with the IMF tilt angle) provides no clear control of the location of FTE signatures.

Citation: Fear, R. C., M. Palmroth, and S. E. Milan (2012), Seasonal and clock angle control of the location of flux transfer event signatures at the magnetopause, J. Geophys. Res., 117, A04202, doi:10.1029/2011JA017235.

\section{Introduction}

[2] Bursts of reconnection at the Earth's magnetopause, called flux transfer events (FTEs), give rise to characteristic signatures that can be observed by spacecraft which are situated near the magnetopause. The clearest signature is a bipolar variation in the component of the magnetic field in the direction that is normal to the local unperturbed magnetopause surface $\left(\mathrm{B}_{N}\right)$, as originally observed by Russell and Elphic [1978]. Perturbations are also observed in the components of the magnetic field tangential to the magnetopause [Paschmann et al., 1982] and plasma signatures that are consistent with magnetopause reconnection are observed [Daly et al., 1984], indicating that magnetic reconnection is the most likely cause of the observational signatures. Statistical studies [e.g., Rijnbeek et al., 1984; Berchem and Russell, 1984] have shown that 'standard polarity' signatures, where the $\mathrm{B}_{N}$ variation is positive then negative, occur mainly in the Northern Hemisphere, whereas 'reverse polarity' signatures (negative then positive) tend to occur in the Southern Hemisphere. If the source region for the signatures is a component reconnection line at low latitudes which tilts in response to the IMF clock angle [Gonzalez and

\footnotetext{
${ }^{1}$ Department of Physics and Astronomy, University of Leicester, Leicester, UK.

${ }^{2}$ Finnish Meteorological Institute, Helsinki, Finland.

Copyright 2012 by the American Geophysical Union. 0148-0227/12/2011JA017235
}

Mozer, 1974] then one might expect that the locations on the magnetopause at which most FTE signatures are observed would depend upon the IMF clock angle, with standard polarity signatures occurring mainly in the postnoon Northern Hemisphere and reverse polarity signatures mainly in the prenoon Southern Hemisphere when $\mathrm{B}_{Y}<0$, and a pattern mirrored about the noon meridian when $\mathrm{B}_{Y}>0$. Although Berchem and Russell [1984] found that the division between the locations of standard and reverse polarity FTEs observed by ISEE was inclined to the GSM equator, the IMF direction did not order the locations of standard and reverse polarity signatures in a simple manner on a case-by-case basis (see Berchem and Russell [1984, Figure 13] and associated discussion). Russell et al. [1985] used the same data set, but combined standard and reverse polarity events (discarding information about the direction in which the FTEs were moving), and combined dawnward IMF orientations with duskward orientations by reflecting the location of FTEs which occurred when the IMF was dawnward about the noon meridian. When this was done, they found that the locations of all FTEs which occurred when the IMF was within $30^{\circ}$ of the GSM equator (with either $\mathrm{B}_{Y}<0$ or $\mathrm{B}_{Y}>0$ ) were aligned in a band parallel to the equator; FTEs which occurred when $\left|B_{Y}\right| \approx\left|B_{Z}\right|$ were aligned in a band which was inclined at about $45^{\circ}$ to the equator, and FTEs which occurred when the IMF was within $30^{\circ}$ of due south were observed over a wide range of latitudes. They concluded that this spatial distribution (ignoring the polarity of the signature) was consistent with a tilted subsolar reconnection line 
[Gonzalez and Mozer, 1974]. Perhaps the strongest evidence for IMF $\mathrm{B}_{Y}$ control of the spatial distribution of FTEs has come from the study of ion anisotropies (an indicator of the hemisphere to which the FTE is connected). Daly et al. [1984] found that when $\mathrm{B}_{Y}>0$, the distribution of events connected to the Northern and Southern Hemispheres was rotated anticlockwise (as viewed looking anti-Sunward) from a strict split between events northward and southward of the equator, and when $\mathrm{B}_{Y}<0$, the distribution was rotated clockwise.

[3] Two of the three major reconnection-based conceptual models proposed to explain the observed signatures invoke a symmetric pair of magnetic structures (a pair of flux tubes in the case of Russell and Elphic [1978], and a pair of magnetopause bulges in the single reconnection line (X line) model proposed by Southwood et al. [1988] and Scholer [1988]). The multiple $\mathrm{X}$ line model [Lee and Fu, 1985] is unusual in its ability to create a single flux rope, when in its simplest form only two X lines are present. In a global MHD simulation using the OpenGGCM model, Raeder [2006] proposed a variant of the Lee and Fu [1985] model: sequential multiple X line reconnection. The Raeder [2006] simulation was based on an interplanetary magnetic field (IMF) with a clock angle of $180^{\circ}$ for one simulation run and $165^{\circ}$ for a second run, and the Earth's dipole tilt was set to $+34^{\circ}$ (equivalent to the tilt at the Northern Hemisphere summer solstice). In both simulation runs, an $\mathrm{X}$ line formed which was southward of the point at which the magnetosheath flow stagnates (i.e., toward the cusp in the winter hemisphere). Subsequently a second X line formed at the stagnation point, and a flux rope was formed between the two X lines. Since the flux rope was southward of the stagnation point, the pressure gradient forces exerted by the magnetosheath flow pushed the flux rope toward the Southern (i.e., winter) Hemisphere. When Raeder [2006] set the dipole tilt to be zero, steady state reconnection occurred, but no flux ropes were formed (i.e., a virtual spacecraft in the simulation would observe no FTE signatures).

[4] Therefore, the Raeder [2006] simulation predicts that near solstice, and when the IMF is directed mainly southward, there should be a tendency for FTE structures to be observed in the winter hemisphere, and when the dipole tilt is small no FTE structures should be observed. (We note that the predicted bias toward the winter hemisphere only applies to FTE signatures observed near the magnetopause; open magnetic field lines still map to the ionosphere in both hemispheres, and so we would still expect ionospheric signatures of bursty reconnection [e.g., Sandholt et al., 1986; Pinnock et al., 1993; Provan et al., 1998; Milan et al., 2000; Wild et al., 2001] in both the winter and summer hemispheres.) However, these effects have not been observed in subsequent simulations. Dorelli and Bhattacharjee [2009] also used the OpenGGCM model to simulate bursty reconnection, but proposed a different mechanism. In their simulation, growth in the magnetopause current density led to a change in the local geometry of the magnetic field which pushed the flow stagnation point away from the subsolar point. The consequent flow shear was unstable to the formation of vortices; the vortices grew and coalesced, and brought together different topological regions of flux (i.e., closed field lines, open field lines connected to the Northern Hemisphere, open field lines connected to the Southern
Hemisphere and magnetosheath field lines) which triggered three dimensional magnetic reconnection within the vortices. Unlike Raeder [2006], Dorelli and Bhattacharjee [2009] found FTEs to be generated when the dipole tilt was zero. (While the Dorelli and Bhattacharjee [2009] simulation was carried out for an IMF clock angle of $135^{\circ}$, they noted that preliminary investigations of the purely southward IMF case led to similar but more complex topologies.) Recently, Tan et al. [2011] used a global-scale hybrid simulation to investigate the effect of dipole tilt. They reported the results of two model runs; the IMF was purely southward in both cases, but the dipole tilt was $+15^{\circ}$ in one case and zero in the other. In the positive dipole tilt case, FTE signatures were observed in both the Northern and Southern Hemispheres. The lack of a bias toward the Southern (winter) Hemisphere appears to contradict the Raeder [2006] results, although we note that the dipole tilt used by Tan et al. [2011] was less than half that used by Raeder [2006]. FTEs were also observed in the zero dipole tilt case, which is inconsistent with Raeder [2006].

[5] Only one observational study has so far looked for a seasonal dependency of in situ FTE signatures. Korotova et al. [2008] examined INTERBALL-1 observations of FTEs during the boreal summers and austral winters in 1995-1999 (which they defined as between days 120-210, i.e., 29 April to 28 July in 1996, and 30 April to 29 July in the nonleap years). During these periods, the INTERBALL spacecraft crossed the prenoon Northern Hemisphere magnetopause, and the prenoon and postnoon Southern Hemisphere magnetopause. FTEs which occurred when the IMF was northward or had only a weak southward component $\left(\mathrm{B}_{Z}>-1 \mathrm{nT}\right)$ were discarded, as they could be due to a highlatitude reconnection site. Of the 69 Southern Hemisphere (i.e., winter hemisphere) magnetopause crossings, 28 had FTE signatures while the lagged IMF $\mathrm{B}_{Z}$ component was southward $\left(\mathrm{B}_{Z}<-1 \mathrm{nT}\right)$; however, none of the 61 Northern (summer) Hemisphere magnetopause crossings had any FTE signatures which occurred when the IMF was southward. Korotova et al. [2008] discussed the possibility that the lack of FTE observations in the Northern Hemisphere could be explained by an unequal distribution of the IMF $\mathrm{B}_{Y}$ component. The component reconnection hypothesis [Gonzalez and Mozer, 1974] predicts that most FTEs in the prenoon Northern Hemisphere would be observed when the IMF is southward and $\mathrm{B}_{Y}>0$. Since the Northern Hemisphere magnetopause crossings (which were all prenoon) exhibited no clear FTE signatures, then the IMF $\mathrm{B}_{Y}$ component would generally have to be negative for a bias in the direction of the IMF to explain the lack of Northern Hemisphere signatures. However, the IMF $\mathrm{B}_{Y}$ component was positive for approximately the same number of Northern Hemisphere magnetopause crossings as it was negative.

[6] In this paper we use a list of flux transfer events observed by Cluster during 2002 and 2003 to examine the presence of any seasonal bias in the FTEs observed by Cluster. The orbit of Cluster is out of phase with that of INTERBALL, and so its orbital bias is different. Cluster observed the near-noon magnetopause in February and March, when the dipole tilt approached zero. The postnoon magnetopause was observed between November and February, when the dipole tilt was negative, and the 


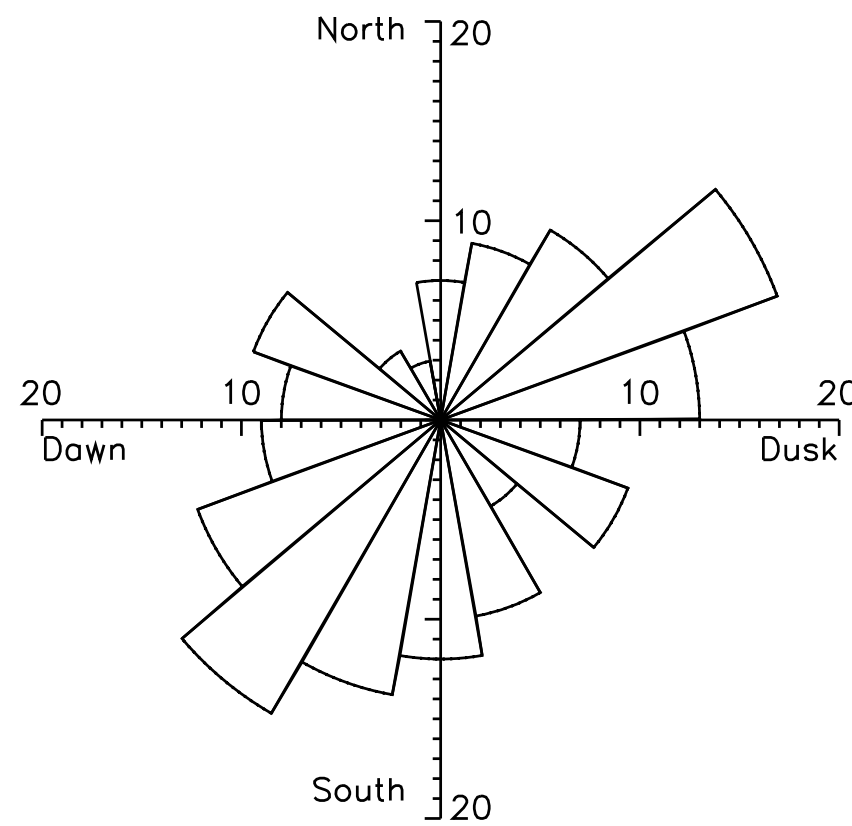

Figure 1. The background IMF GSM $\mathrm{B}_{Y}$ and $\mathrm{B}_{Z}$ distribution for November 2002 through June 2003, measured by taking the lagged IMF at the time of each magnetopause crossing made by Cluster during this time frame (reproduced from Fear et al. [2007]).

prenoon magnetopause was observed between March and June, when the dipole tilt was mostly positive. In section 2, we discuss the FTE data set that is used in this paper. We then examine the dependence of the FTE location on the IMF clock angle and geomagnetic dipole tilt. We compare the observations with the seasonal bias predicted by Raeder [2006] and briefly consider the dependence on the effective dipole tilt (which combines the geomagnetic dipole tilt and the IMF tilt angle in the GSM X-Z plane) in the discussion section, and then summarize our conclusions.

\section{Data Set and Instrumentation}

[7] We use a list of 446 FTEs observed by Cluster between November 2002 and June 2003. During these months, the typical separation of the spacecraft was of the order of $5000 \mathrm{~km}$. Due to the nature of Cluster's orbit in 2002 and 2003, the FTEs were observed at high northern and southern latitudes near local noon, and at lower latitudes on the magnetopause flanks. The list of events was originally compiled by Fear et al. [2005], who discussed the origin and motion of the subset of FTEs which occurred in the postterminator region when the IMF was within $70^{\circ}$ of north. Further statistical results have considered the velocity and plasma signatures of FTEs for all IMF conditions [Fear et al., 2007] and compared the degree of asymmetry in the observed $\mathrm{B}_{N}$ signatures with 2D MHD predictions for single and multiple $X$ line scenarios [Fear et al., 2010]. However, none of the previous studies have examined the effect of dipole tilt or the dependence of the location of the FTE signatures upon the IMF $\mathrm{B}_{Y}$ component.

[8] The FTEs were originally identified by Fear et al. [2005] if a clear bipolar $\mathrm{B}_{N}$ signature was observed by the
Cluster FGM instrument [Balogh et al., 2001] at one or more of the Cluster spacecraft, coupled with an enhancement or decrease (a 'crater' signature) in the magnitude of the magnetic field. In other words, signatures observed nearly simultaneously at several of the Cluster spacecraft were only classified as a single FTE. The event catalog has not previously been published, so it is included in the auxiliary material to this paper. ${ }^{1}$

[9] We use the lagged IMF values for each event that were determined by Fear et al. [2005], based on the interplanetary magnetic field and solar wind velocity observations provided by the ACE MAG [Smith et al., 1998] and SWEPAM [McComas et al., 1998] instruments.

[10] In section 3, we will refer to the clock angle as a convenient means of dividing the data. The clock angle is an angle in the GSM Y-Z plane. We will use this angle both to refer to the lagged IMF (the IMF clock angle):

$$
\theta_{B}=\arctan \left[B_{Y} / B_{Z}\right],
$$

and to a location at which an FTE signature is observed, projected onto the GSM Y-Z plane (the location clock angle):

$$
\theta_{L}=\arctan \left[Y_{G S M} / Z_{G S M}\right]
$$

For this study, we consider only the 300 FTEs which occurred when the lagged IMF clock angle $\theta_{B}$ was between $70^{\circ}$ and $290^{\circ}$.

[11] It is important to note from the outset that there is an IMF $\mathrm{B}_{Y}$ bias in our data. Figure 1 shows the lagged IMF clock angle evaluated at each magnetopause crossing made by Cluster between November 2002 and June 2003. It is evident from Figure 1 that the most likely IMF configurations are northward/duskward and southward/dawnward. Therefore, when considering only FTEs which occur when the IMF has a southward component, $\mathrm{B}_{Y}$ is more likely to be negative than positive.

\section{Dipole Tilt and IMF Clock Angle}

[12] Figure 2 shows the data used in this study, color coded by the dipole tilt angle at the time of each observation. In addition to the bias in the IMF distribution noted above, there are two sources of orbital bias to which we draw attention. First, since the orbit of Cluster crossed the dusk flank magnetopause in November and December 2002, the noon-sector magnetopause in February and March 2003 and the dawn flank in May and June 2003, the dipole tilt was negative when the spacecraft crossed the magnetopause at $Y \gtrsim 0$, and positive when the spacecraft crossed the magnetopause at $\mathrm{Y} \lesssim 0$. Secondly, the spacecraft crossed the magnetosphere at higher latitudes in the Southern Hemisphere than in the north. Consequently, the distribution of signatures is not symmetric about the GSM equator: the signatures observed in the Northern Hemisphere all occur equatorward of $Z_{G S M} \approx 10 \mathrm{R}_{E}$, whereas many of the Southern Hemisphere events occur poleward of $Z_{G S M} \approx-10 \mathrm{R}_{E}$.

[13] Rather than a simple north-south divide between the standard and reverse polarity signatures, the division is rotated clockwise such that fewer standard polarity

\footnotetext{
${ }^{1}$ Auxiliary materials are available at $\mathrm{ftp} / / \mathrm{ftp}$.agu.org/apend/ja/ $2011 \mathrm{ja} 017235$.
} 

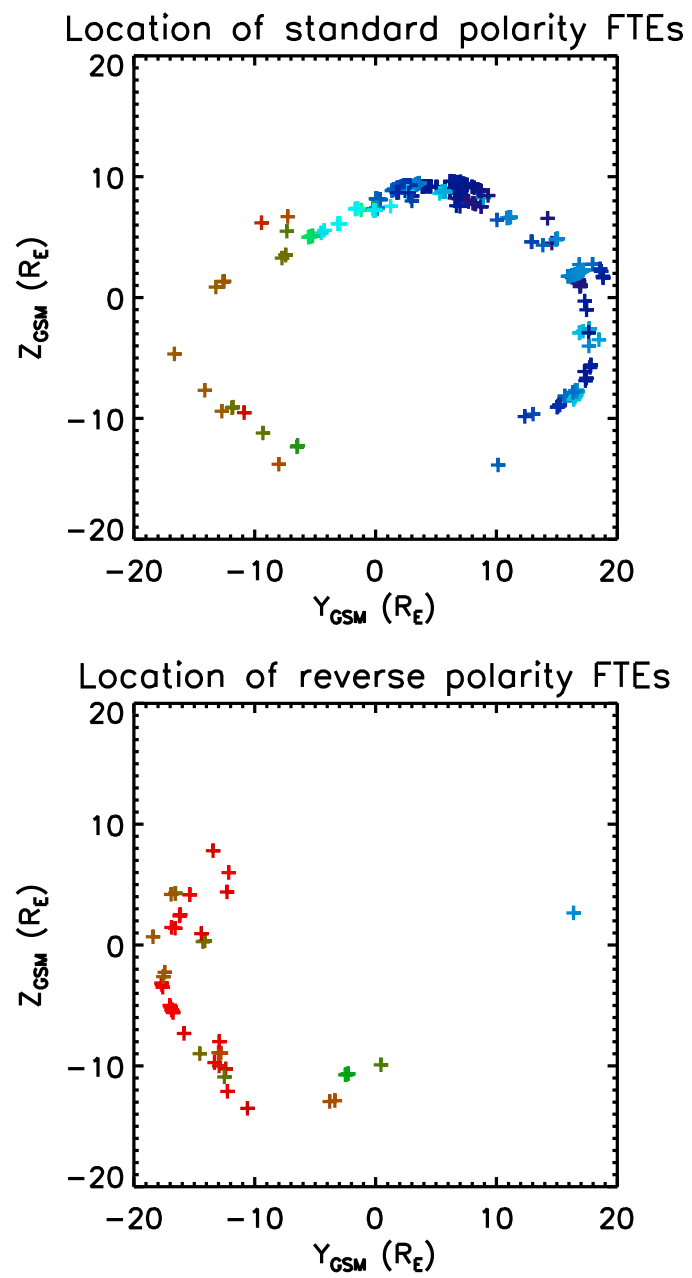

Figure 2. The location of the (top) standard and (bottom) reverse polarity FTE signatures used in this study, color coded by the geomagnetic dipole tilt. The magnetopause crossings on the dusk flank occurred in November and December 2002 (negative tilt) and the crossings on the dawn flank occurred in May and June 2003 (positive tilt).

signatures are observed in the northern/prenoon quadrant, and more are observed in the southern/postnoon quadrant (Figure 2). Similarly, the reverse polarity signatures are observed almost exclusively in the southern/prenoon quadrant, with some events also observed in the northern/prenoon quadrant. This rotation is consistent with a source region being a component reconnection line passing through or near the subsolar point, as Figure 1 shows that a southward and dawnward IMF orientation occurred more frequently than a southward and duskward orientation.

[14] To investigate the IMF clock angle dependence, we split the events into three groups, which are shown in Table 1. In order to maintain a reasonable number of FTEs in each IMF clock angle bin, we have selected bins which are broadly equal in angular range. The southward IMF bin is therefore broader than the range of clock angles used by Raeder [2006], but note that it is also the least populous bin in terms of the number of FTE signatures that were observed when the lagged IMF clock angle was within this range; reducing the size of the clock angle bin would weaken the statistics. In this section, we will discuss the variation of the FTE locations on IMF clock angle, and how they are affected by orbital bias and a possible seasonal effect.

\subsection{Southward IMF}

[15] Figure 3 shows the distribution of FTE signatures and magnetopause crossings when the IMF was southward $\left(145^{\circ}\right.$ $<\theta_{B}<215^{\circ}$, indicated at top left). Figure 3a shows the location of all magnetopause crossings made when $\theta_{B}$ was between $145^{\circ}$ and $215^{\circ}$ at the time of the crossing. The Northern Hemisphere crossings are regularly distributed between $\mathrm{Y}_{G S M} \pm 15 \mathrm{R}_{E}$. Figure $3 \mathrm{~b}$ shows the location of the magnetopause crossings for which $\theta_{B}$ was between $145^{\circ}$ and $215^{\circ}$ at the time of the crossing, but on which no clear FTE signatures were observed. The Northern Hemisphere crossings with no clear FTEs are mostly distributed in the prenoon quadrant: 16 prenoon Northern Hemisphere magnetopause crossings occur with the southward IMF criterion, 11 of which had no clear FTE signatures, whereas only 2 of the 12 postnoon Northern Hemisphere magnetopause crossings had no clear FTE signatures.

[16] The IMF can of course change its orientation between the time a spacecraft crosses the magnetopause and the time it observes an FTE signature. Figures $3 \mathrm{c}$ and $3 \mathrm{~d}$ show the location of each standard and reverse polarity signature which occurred when $\theta_{B}$ was between $145^{\circ}$ and $215^{\circ}$ at the time the FTE signature was observed. The same data are represented as polar histograms in Figures 3e and 3f, where the length of each wedge represents the number events which occurred in each location clock angle bin. The average location of the standard and reverse polarity signatures is indicated by a red arrow which represents the directional mean, calculated as described by Mardia and Jupp [2000, p. 15]. The location of the $j$ th FTE in the GSM Y/Z plane was represented by a unit vector:

$$
\mathbf{x}_{j}=\left(\sin \theta_{L j}, \cos \theta_{L j}\right) .
$$

(The distance from the subsolar point at which the FTE was observed was ignored, as this is determined by the intersection of the orbit of the spacecraft with the magnetopause.) The mean direction vector is calculated by determining the center of mass of the signature locations $(\bar{S}, \bar{C})$, where:

$$
\begin{gathered}
\bar{S}=\frac{1}{n} \sum_{j=1}^{n} \sin \theta_{L j} \\
\bar{C}=\frac{1}{n} \sum_{j=1}^{n} \cos \theta_{L j} .
\end{gathered}
$$

Table 1. The IMF Clock Angle Divisions Used and the Number of Standard and Reverse Polarity Signatures Which Occurred When the Lagged IMF Clock Angle $\left(\theta_{B}\right)$ was in Each Range

\begin{tabular}{ccc}
\hline$\theta_{B}$ Range & $\begin{array}{c}\text { Number of Standard } \\
\text { Signatures }\end{array}$ & $\begin{array}{c}\text { Number of Reverse } \\
\text { Signatures }\end{array}$ \\
\hline $70^{\circ}<\theta_{B}<145^{\circ}$ & 84 & 14 \\
$145^{\circ}<\theta_{B}<215^{\circ}$ & 29 & 7 \\
$215^{\circ}<\theta_{B}<290^{\circ}$ & 138 & 27 \\
\hline
\end{tabular}



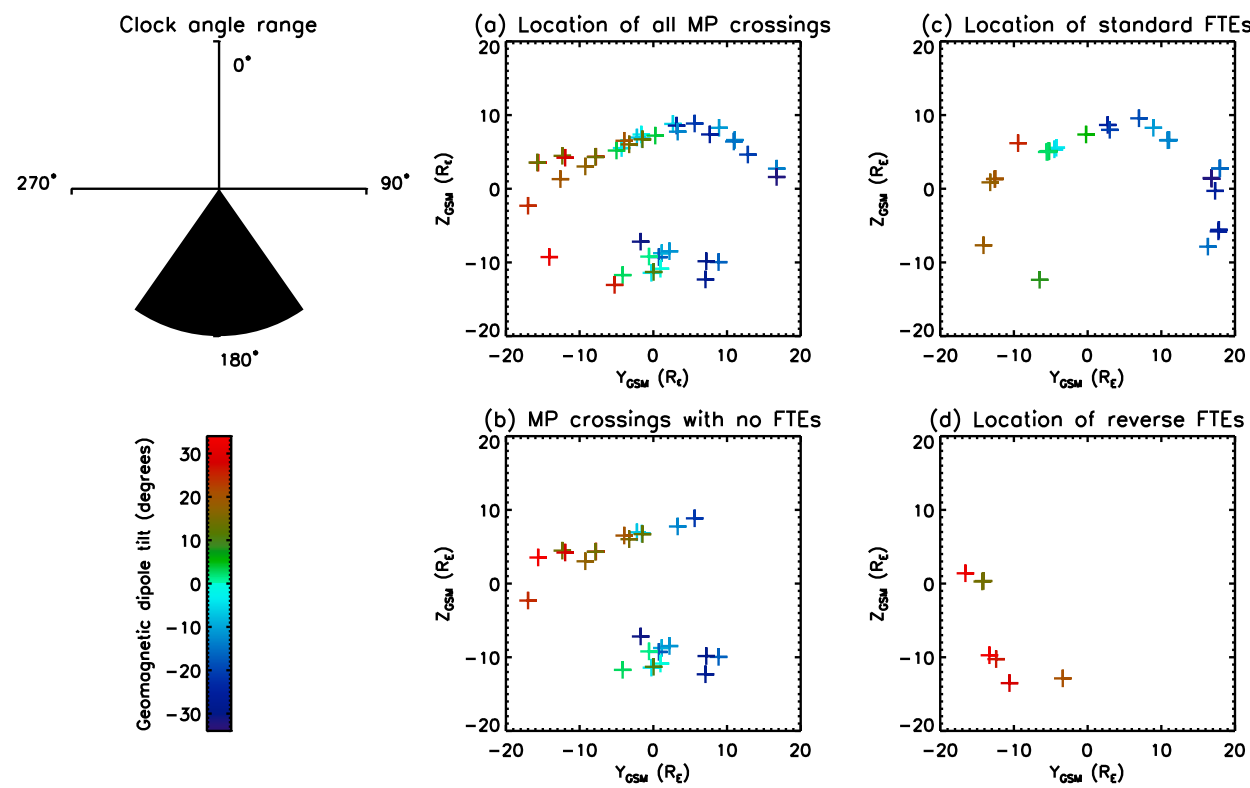

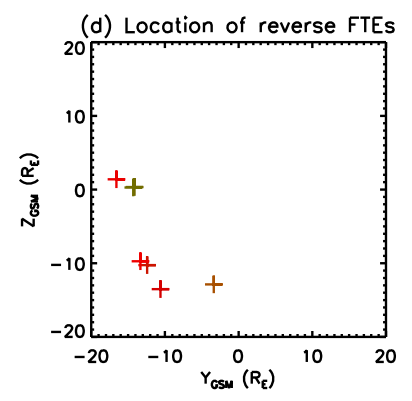

(e) Locotion of stondard FTEs

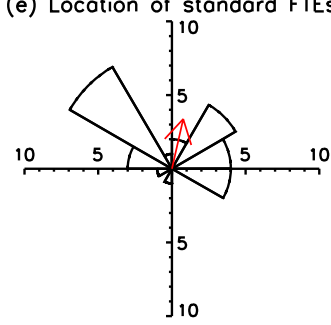

(f) Location of reverse FTEs

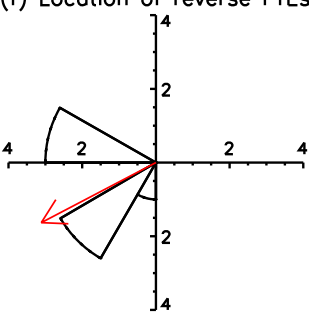

Figure 3. The location of the flux transfer events identified by Fear et al. [2005, 2007], which occurred when (top left) the IMF clock angle was between $145^{\circ}$ and $215^{\circ}$. (a) The location of all magnetopause crossings made by Cluster for which the lagged IMF was between $145^{\circ}$ and $215^{\circ}$ at the time of the magnetopause crossing. (b) The subset of the magnetopause crossings on which no clear FTE signatures were observed. The locations at which the (c) standard and (d) reverse polarity signatures were observed. Polar histograms indicating the locations (in clock angle) of (e) standard and (f) reverse polarity signatures within the current clock angle range. The red arrow denotes the directional mean, which is scaled such that a vector with a length equal to the distance from the origin to the end of the axis has a magnitude of 1 . In Figures 3a-3d, the magnetopause and FTE locations are color coded by the geomagnetic dipole tilt at the time of each magnetopause crossing or FTE observation.

The mean direction $(\bar{S}, \bar{C})$ is a vector with length:

$$
\bar{R}=\sqrt{\bar{S}^{2}+\bar{C}^{2}}
$$

and a direction given by a clock angle:

$$
\bar{\theta}=\arctan (\bar{S} / \bar{C}) .
$$

The mean direction vectors plotted in Figures $3 \mathrm{e}$ and $3 \mathrm{f}$ are scaled such that a vector with a length equal to the distance from the origin to the end of the axis has a magnitude of $\bar{R}=1$. Since all of the vectors contributing to a mean vector have unit magnitude, if they all lie in the same direction then $\bar{R}$ will also be one. If the vectors are less closely grouped, the magnitude will decrease. Therefore, the length of the mean direction vector relative to the axis length is an indication of how tightly grouped the contributing vectors (the FTE locations) are, and it is also an indicator of the significance of the mean direction.

[17] When the IMF is within $35^{\circ}$ of due south, the standard polarity signatures are distributed throughout the Northern Hemisphere (Figures $3 \mathrm{c}$ and 3e), with no pattern immediately evident. The lack of a significant peak in the directional distribution is also indicated by the short mean direction vector in Figure 3e. However, the majority of the standard polarity signatures observed in the northern prenoon quadrant occur when the dipole tilt is relatively small. Figure 4 shows the same data as Figure 3, but the magnetopause crossings and FTE signatures which occurred when the geomagnetic dipole tilt was between $\pm 6^{\circ}$ have been removed. If the Raeder [2006] predictions are correct, then there should be little difference between Figures 3 and 4 as FTEs would not be generated when the dipole tilt was small. However, there are eight FTEs in the southward IMF bin which occurred when the magnitude of the geomagnetic dipole tilt was less than $6^{\circ}$. All eight events occurred when there was a significant IMF $\mathrm{B}_{X}$ component; the IMF tilt angle, ignoring the sign of $\mathrm{B}_{X}\left(\arctan \left(\left|B_{X}\right| / B_{Z}\right)\right)$ was less than $155^{\circ}$ in all eight cases, indicating that they were more than $25^{\circ}$ from the $180^{\circ}$ IMF tilt angle $\left(B_{X}=0\right)$ cases that were simulated by Raeder [2006]. Once these near-equinox FTEs and magnetopause crossings are removed, it is evident that most of the FTE signatures occur in the northern postnoon quadrant (Figures 4c and 4e), and most of the Northern Hemisphere magnetopause crossings with no clear FTE signatures occur prenoon (Figure 4b). A small number of standard polarity FTEs are present in the prenoon Northern Hemisphere, when the dipole tilt is positive, but these are a small proportion of the total number of standard polarity FTE signatures observed in the dominantly southward IMF bin, and we emphasize that most of the prenoon Northern Hemisphere magnetopause crossings are devoid of clear FTE signatures. Therefore, although the standard polarity signatures in the prenoon Northern Hemisphere are not consistent with a strict application of the Raeder [2006] predictions, the seasonal effect predicted by Raeder [2006] appears to be present as an overall trend. 

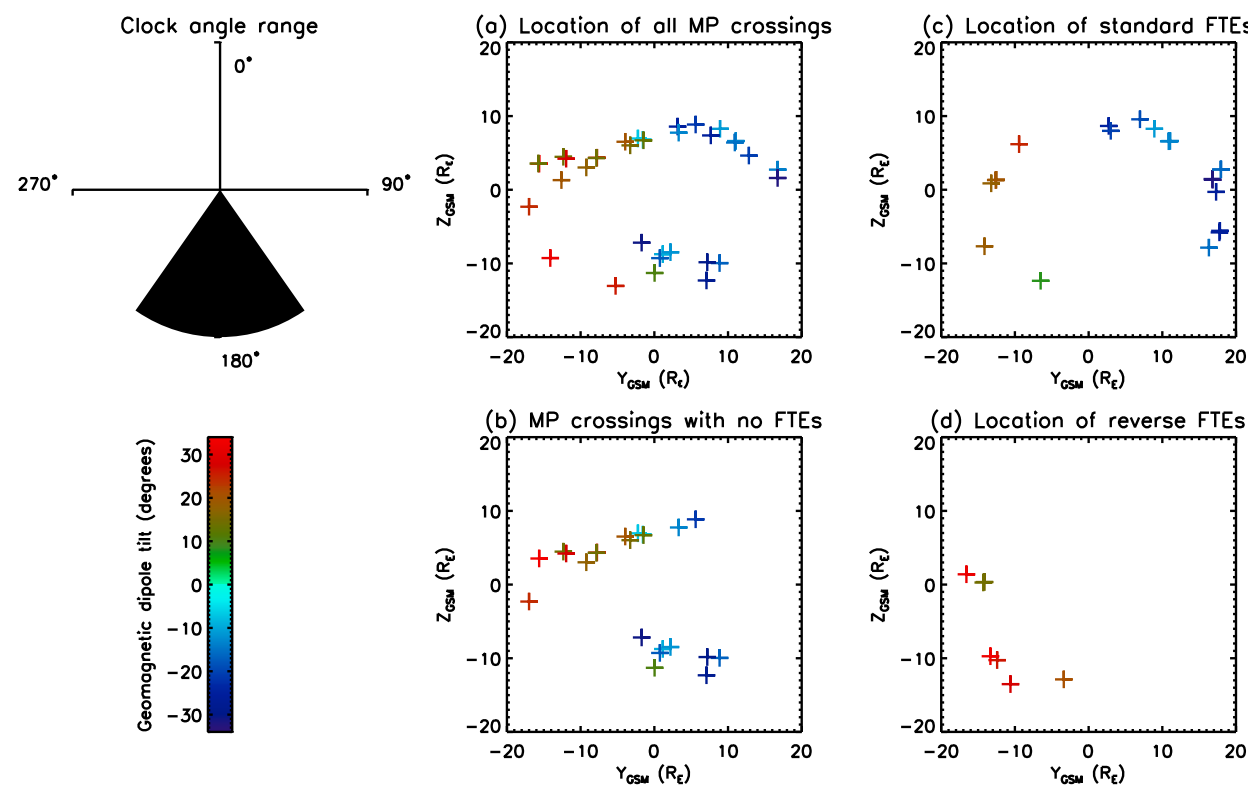

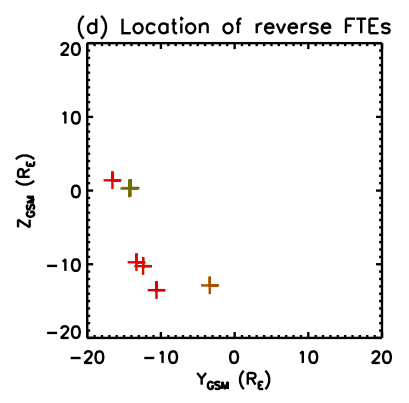

(e) Locotion of stondard FTEs

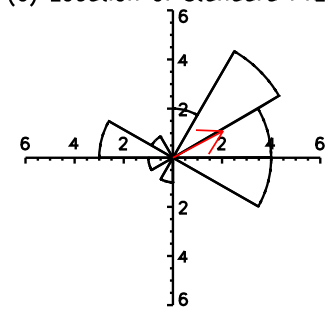

(f) Location of reverse FTEs

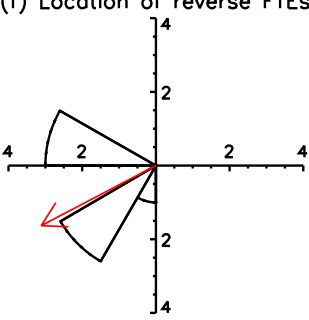

Figure 4. The location of the flux transfer events and magnetopause crossings which occurred when the IMF clock angle was between $145^{\circ}$ and $215^{\circ}$ and where the magnitude of the dipole tilt was greater than $6^{\circ}$. Figure 4 follows the same format as Figure 3.

[18] There are far fewer reverse polarity signatures than standard polarity signatures in this clock angle range (Figure 3d). This is true in general in our data set, and is partly because the Southern Hemisphere magnetopause crossings made by Cluster in 2002 and 2003 occurred at higher latitudes than their Northern Hemisphere counterparts. It is possible that FTEs may dissipate in the cusp [e.g., Omidi and Sibeck, 2007], and as the Southern Hemisphere magnetopause crossings made by Cluster in 2002 and 2003 occurred at higher GSM latitudes than the Northern Hemisphere crossings, they were more likely to occur through the cusp, or poleward of the cusp, which explains the large number of magnetopause crossings without FTE signatures between $\mathrm{Y}_{G S M} \pm 5 \mathrm{R}_{E}$ and at $\mathrm{Z}_{G S M} \approx-10 \mathrm{R}_{E}$ (Figure $3 \mathrm{~b}$ ). The reverse polarity signatures which are observed within this IMF clock angle bin all occurred in the prenoon hemisphere, either in the Southern Hemisphere or just northward of the GSM equator, during the Southern Hemisphere winter (indicated by the red and brown color coding, which represents positive dipole tilt). Three postnoon Southern Hemisphere magnetopause crossings were made away from the cusp region $\left(\mathrm{Y}_{G S M}>5 \mathrm{R}_{E}\right)$; all three were crossings on which no clear FTE signatures were observed (Figure 3a and $3 b)$.

[19] The observations in Figure 4 are summarized in Table 2, with reference to zones on the magnetopause (equivalently ranges of $\theta_{L}$ ) that are shown in Figure 5 . Ignoring a dipole tilt effect but assuming a component reconnection site which is aligned with the GSM equator, when the IMF is exactly southward one would expect to observe standard polarity signatures in zones (7), (8), (1), and (2), and reverse polarity signatures in zones (3)-(6). In practice, $\mathrm{B}_{Y}$ is unlikely to be exactly zero, and the clock angle bins we have selected are quite broad; if $\mathrm{B}_{Y}<0$ then we would expect to see some reverse polarity signatures in (7) and some standard polarity signatures in (3). Likewise, if
$\mathrm{B}_{Y}>0$ then we would expect some standard polarity signatures in (6) and some reverse polarity signatures in (2).

[20] However, if the predicted seasonal bias is present, then we expect to observe no standard polarity FTE signatures in zones (6)-(8) and no reverse polarity signatures in zones (2)-(4) (since Cluster would only observe these polarity-zone combinations when the spacecraft are on 'summer' side of the reconnection line). Therefore, for the IMF clock angle range considered in Figure 4, and after removing near-equinox events, we expect standard polarity signatures in zones (1) and (2) and reverse polarity signatures in (5) and (6) for both positive and negative $\mathrm{B}_{Y}$ (but

Table 2. Summary Table for Figure $4^{\mathrm{a}}$

\begin{tabular}{lccccc}
\hline & & \multicolumn{2}{c}{ 'Positives' } & & 'Negatives' \\
\cline { 3 - 4 } \cline { 5 - 6 } & Prediction & $\begin{array}{c}\text { Standard } \\
\text { Signatures }\end{array}$ & $\begin{array}{c}\text { Reverse } \\
\text { Signatures }\end{array}$ & & $\begin{array}{c}\text { Crossings With } \\
\text { No Signatures }\end{array}$ \\
\hline Zone 1 & Standard & 3 & 0 & 2 \\
Zone 2 & Standard & 8 & 0 & 0 \\
Zone 3 & Standard & $4^{\text {c }}$ & 0 & 0 \\
Zone 4 & None & 0 & 0 & 7 \\
Zone 5 & Reverse & 1 & 2 & 1 \\
Zone 6 & Reverse & 1 & 2 & 6 \\
Zone 7 & Reverse & 4 & $3^{\mathrm{c}}$ & \\
Zone 8 & None & 0 & 0 & 5 \\
Total & & 21 & 7 & 22
\end{tabular}

${ }^{\mathrm{a}}$ The zone number refers to the portion of the magnetopause (projected into the GSM Y-Z plane) indicated in Figure 5. The prediction column details whether the combined IMF control and seasonal effect predicts the presence of standard or reverse polarity FTE signatures or no FTE signatures. The remaining columns detail, for each zone, the total number of standard and reverse polarity signatures and the number of magnetopause crossings on which no FTE signatures were observed (all for $145^{\circ}<\theta_{B}<215^{\circ}$ ). 'Positives' refers to the presence of FTE signatures and 'negatives' refers to the absence of FTE signatures.

${ }^{\mathrm{b}}$ Only when $\mathrm{B}_{Y}<0$.

${ }^{\mathrm{c}}$ All occur when $\mathrm{B}_{Y}<0$. 


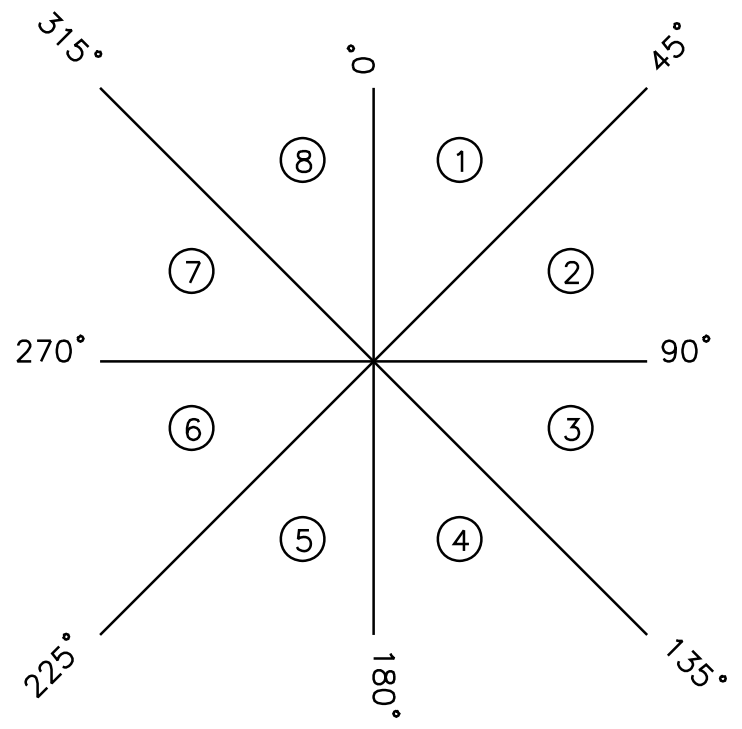

Figure 5. The zones used in the summary tables in Tables 2, 4, and 6. The location clock angle, $\theta_{L}$, is labeled around the edge and each zone is numbered. Cluster crossed the magnetopause in zones 1-4 when the geomagnetic dipole tilt was negative and zones 5-8 when the tilt was positive.

where the IMF is still dominated by its negative $\mathrm{B}_{Z}$ component). When $\mathrm{B}_{Y}$ is slightly negative, we also expect to see some standard polarity signatures in zone (3) and reverse polarity signatures in zone (7). Since, in the absence of a seasonal effect, only reverse polarity signatures are predicted in zone (4) and only standard polarity signatures are expected in zone (8), but both zones are observed during local summer, then no signatures are expected in these zones. (We also expect the seasonal bias effect, if present, to suppress the standard polarity signatures in (6) and reverse polarity signatures in (2) that might occur when $\mathrm{B}_{Y}$ is slightly positive.) These predictions, along with the number of standard and reverse polarity signatures and magnetopause crossings with no FTE signatures which occurred in each zone when $\theta_{B}$ was between $145^{\circ}$ and $215^{\circ}$, are listed in Table 2 .

[21] The data in Table 2 are further distilled in a basic truth table in Table 3. FTE signatures are referred to as 'positives'. A 'true positive' occurs when an FTE is observed with the correct polarity in a zone in which it is predicted (i.e., standard polarity signatures in zones (1)-(3) and reverse polarity signatures in zones (5)-(7)). (Since all four standard polarity signatures in zone (3) and all three reverse polarity signatures in zone (7) occurred when the lagged IMF was negative, they are counted as true positives.) A false positive occurs when an FTE signature is observed in a zone in which no FTEs are predicted, or if the polarity of the signature is contrary to the prediction. (e.g., The six false positives in Table 3 are made up of the standard polarity signatures in zones (5)-(7), and would include reverse polarity signatures in (1)-(3) or any signatures in (4) and (8) if any were present.) The true positives outnumber the false positives by a ratio of more than three to one.

[22] Magnetopause crossings without clear FTE signatures are referred to as 'negatives'. Those in zones (4) and (8), where no FTEs are predicted, are 'true negatives', whereas those in zones where signatures are predicted are labeled 'false negatives'. We use this terminology for simplicity, but note a major caveat that 'false negatives' are not inconsistent with the combined component reconnection/seasonal bias prediction: other factors affect the occurrence of reconnection [e.g., Phan et al., 2010, and references therein], and we do not suggest that reconnection must occur and FTE signatures must be observed whenever the location of the spacecraft and dipole tilt is favorable. Nonetheless, we find that the number of true negatives does significantly outnumber the number of 'false' negatives.

\subsection{Dawnward IMF}

[23] When we consider a significant negative $\mathrm{B}_{Y}$ component (Figure 6), it is evident that the distribution of standard and reverse polarity signatures is rotated clockwise from a strict north-south split, as would be expected from a rotation of a component X line [Gonzalez and Mozer, 1974] which would tilt from the top-left to the bottom-right quadrants in Figure $6 \mathrm{c}$ and $6 \mathrm{~d}$. The standard polarity signatures are mostly distributed in the postnoon Northern Hemisphere, with a significant number of events occurring southward of the equator, postnoon. The reverse polarity signatures in Figure 6d and $6 \mathrm{f}$ occur almost exclusively prenoon, with several events occurring in the Northern Hemisphere (prenoon). Only one Northern Hemisphere postnoon magnetopause crossing contained no clear FTE signatures (Figure 6b), and only three of the seven Southern Hemisphere prenoon magnetopause crossings which occurred away from the cusp region $\left(\mathrm{Y}_{G S M}<-5 \mathrm{R}_{E}\right)$ had no clear FTE signatures.

[24] The data in Figure 6 are summarized in tabular form in Tables 4 and 5, which take the same form as Tables 2 and 3. Since the IMF is dawnward, we would expect a clockwise rotation of the reconnection line, with standard polarity signatures above and to the right, and reverse polarity signatures below and to the left. However, the seasonal bias should still apply; therefore crudely speaking, we would expect standard polarity signatures in zones (1)-(3), reverse polarity signatures in zones (5)-(7), and no signatures in zones (4) and (8). The number of true positives (signatures in zones where they are predicted, and with consistent

Table 3. Truth Table for Figure $4^{\mathrm{a}}$

\begin{tabular}{lcc}
\hline & $\begin{array}{c}\text { Positives } \\
\text { (Number of Signatures) }\end{array}$ & $\begin{array}{c}\text { Negatives } \\
\text { (Number of Crossings) }\end{array}$ \\
\hline True & 22 & 18 \\
False & 6 & 4 \\
Total & 28 & 22 \\
\hline
\end{tabular}

aThis table summarizes the numbers in Table 2. The 'true positives' cell gives the total number of standard and reverse polarity signatures observed in zones which were predicted to contain them (with the correct polarity) and the 'true negatives' cell gives the number of magnetopause crossings without FTE signatures which occurred in zones in which no signatures are predicted. The 'false positives' cell gives the total number of FTEs observed in zones which were predicted to have no FTEs plus the total number of FTE signatures which had a polarity which was opposite to that predicted for the zone and the 'false negatives' cell gives the number of magnetopause crossings without FTE signatures which occurred in zones where FTE signatures were predicted. We use the term 'false negatives' for simplicity, but a magnetopause crossing without FTE signatures even though the IMF and spacecraft location are favorable to their observation is not necessarily inconsistent with the predictions. 

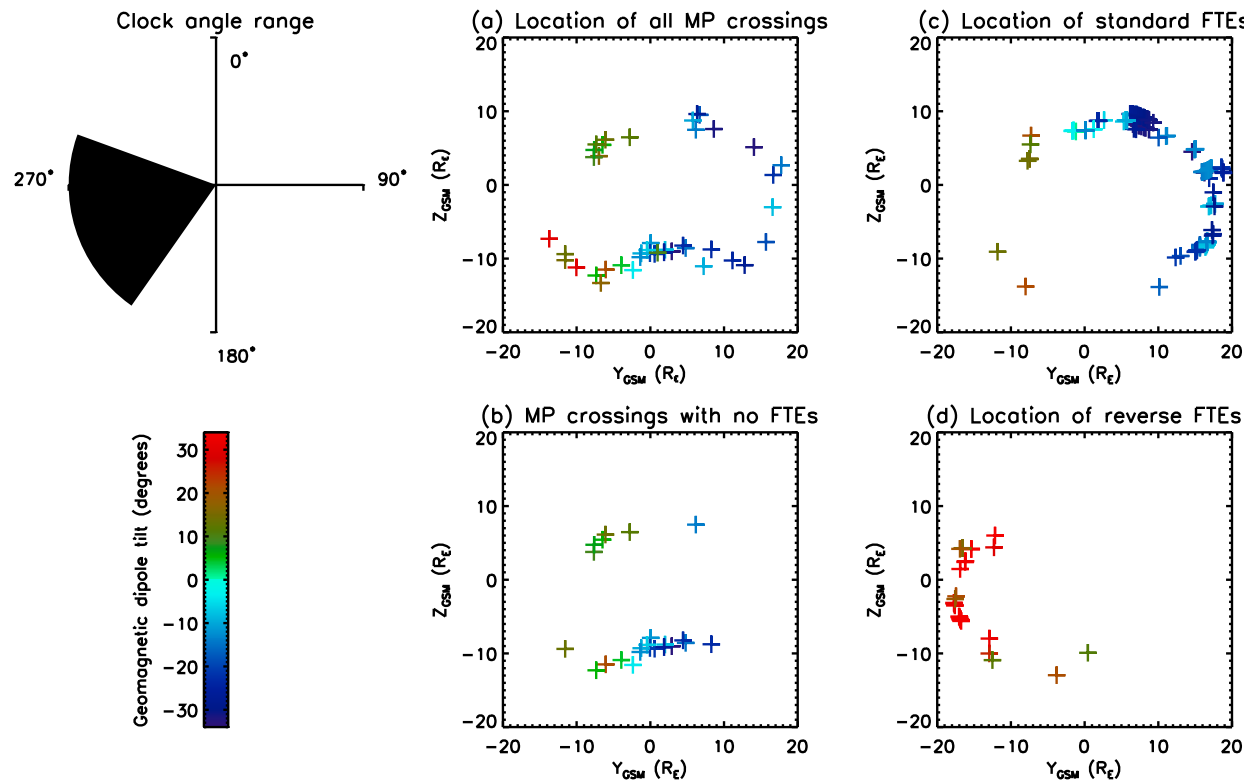

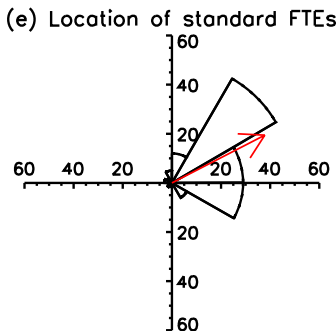

(f) Location of reverse FTEs

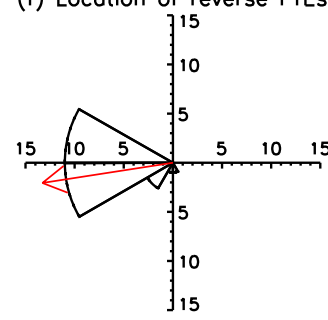

Figure 6. The location of the flux transfer events and magnetopause crossings which occurred when the IMF clock angle was between $215^{\circ}$ and $290^{\circ}$. Figure 6 follows the same format as Figure 3.

polarities) again outnumber the false positives (signatures with the 'wrong' polarity, or in zones where no signatures are predicted), this time by a factor of nearly eleven to one. The number of 'false' negatives is slightly larger than the number of true negatives. As noted above, a 'false' negative is not actually inconsistent with the predictions, but the majority occur in zone (5); the magnetopause crossings in this zone are most likely to be through or poleward of the cusp.

\subsection{Duskward IMF}

[25] When there is a significant positive $\mathrm{B}_{Y}$ component (Figure 7), one would expect the distribution of standard and reverse polarity signatures to be rotated anticlockwise (consistent with a tilted $\mathrm{X}$ line which extends from the top-right to bottom-left quadrants in Figure 7c and 7d). There are two features in Figure $7 \mathrm{c}$ which hint at such a rotation. First, more standard polarity FTE signatures are indeed observed in the southern/prenoon quadrant than in the southward or dawnward IMF cases (Figures 3c and 6c), although they are a small fraction of the total number of standard polarity

Table 4. Summary Table for Figure $6^{\text {a }}$

\begin{tabular}{|c|c|c|c|c|}
\hline & \multirow[b]{2}{*}{ Prediction } & \multicolumn{2}{|c|}{ 'Positives' } & \multirow{2}{*}{$\begin{array}{c}\text { 'Negatives' } \\
\text { Crossings With } \\
\text { No Signatures }\end{array}$} \\
\hline & & $\begin{array}{c}\text { Standard } \\
\text { Signatures }\end{array}$ & $\begin{array}{c}\text { Reverse } \\
\text { Signatures }\end{array}$ & \\
\hline Zone 1 & Standard & 52 & 0 & 1 \\
\hline Zone 2 & Standard & 38 & 0 & 0 \\
\hline Zone 3 & Standard & 35 & 0 & 0 \\
\hline Zone 4 & None & 1 & 1 & 8 \\
\hline Zone 5 & Reverse & 1 & 1 & 8 \\
\hline Zone 6 & Reverse & 1 & 14 & 1 \\
\hline Zone 7 & Reverse & 5 & 11 & 3 \\
\hline Zone 8 & None & 5 & 0 & 2 \\
\hline Total & & 138 & 27 & 23 \\
\hline
\end{tabular}

${ }^{\mathrm{a}}$ Same format as Table 2 . signatures observed in this clock angle bin. Secondly, fewer standard polarity signatures are observed in the southern/ postnoon quadrant compared with dawnward IMF orientations. However, the reverse polarity signatures are still concentrated in the southern prenoon quadrant, rather than the southern postnoon quadrant as would be expected if Figure 7 were a mirror image of Figure 6, and there is a lack of standard polarity signatures in the prenoon Northern Hemisphere (Figure 7c) notwithstanding the fact that Cluster did cross the magnetopause in this region when the IMF had a significant positive $\mathrm{B}_{Y}$ component (Figure $7 \mathrm{~b}$ ). Nonetheless, comparing the central peaks and mean directions of the histograms with the southward and dawnward IMF cases (Figure $7 \mathrm{e}$ with Figures $4 \mathrm{e}$ and $6 \mathrm{e}$, and Figure $7 \mathrm{f}$ with Figures $4 \mathrm{f}$ and $6 \mathrm{f}$ ), it is evident that the peaks in the distributions have rotated anticlockwise relative to the other two cases, although they are rotated clockwise relative to the vertical axis.

[26] The facts that only a few standard polarity signatures are observed in the northern prenoon quadrant, and no reverse polarity signatures in the southern postnoon quadrant, are both consistent with the seasonal bias. The data in Figure 7 are summarized in Tables 6 and 7. This time, since the IMF is duskward, we would expect an anticlockwise rotation of the reconnection line. When combined with the seasonal bias, we expect standard polarity signatures in zone (1), reverse polarity signatures in zone (5), and no signatures in zones (2)-(4) and (6)-(8). The true positives again

Table 5. Truth Table for Figure $6^{\mathrm{a}}$

\begin{tabular}{lcc}
\hline & $\begin{array}{c}\text { Positives } \\
\text { (Number of Signatures) }\end{array}$ & $\begin{array}{c}\text { Negatives } \\
\text { (Number of Crossings) }\end{array}$ \\
\hline True & 151 & 10 \\
False & 14 & 13 \\
Total & 165 & 23 \\
\hline
\end{tabular}

\footnotetext{
${ }^{\mathrm{a}} \mathrm{Same}$ format as Table 3 .
} 

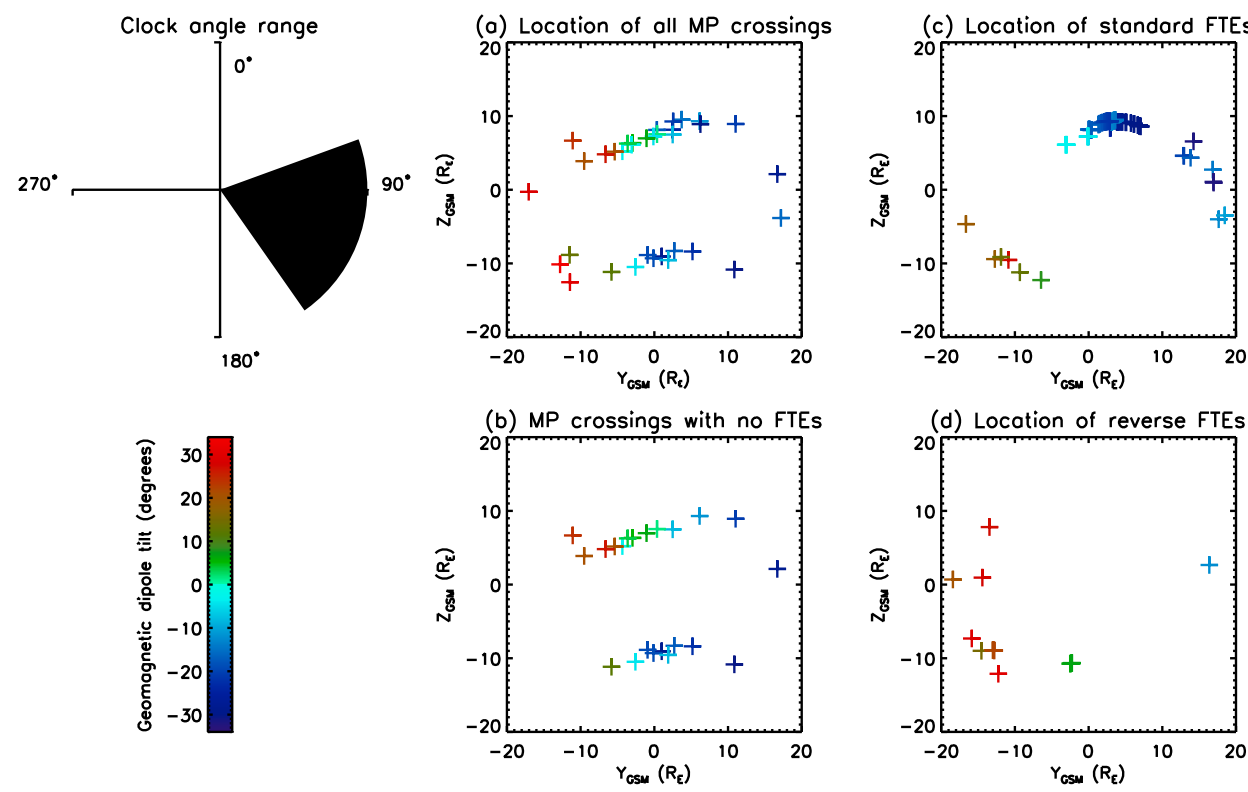

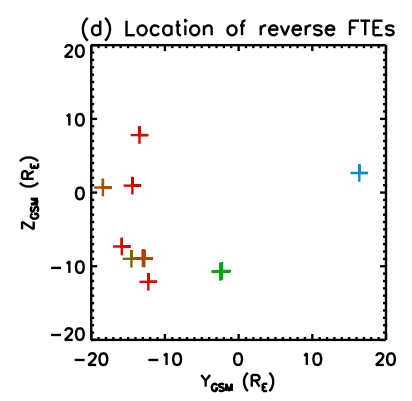

(e) Locotion of stondard FTEs

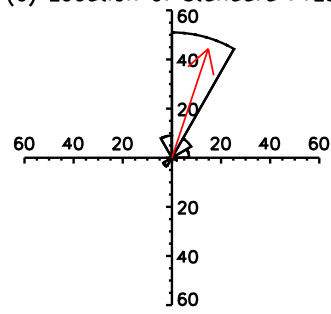

(f) Location of reverse FTEs

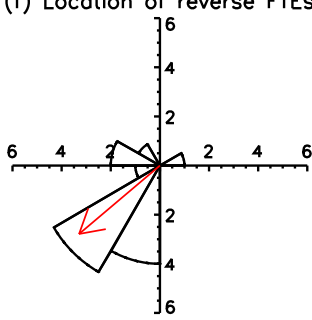

Figure 7. The location of the flux transfer events and magnetopause crossings which occurred when the IMF clock angle was between $70^{\circ}$ and $145^{\circ}$. Figure 7 follows the same format as Figure 3.

outnumber the false positives by nearly two to one, and the same is true of the true and 'false' negatives.

\section{Discussion}

\subsection{Dipole Tilt: Seasonal Effect}

[27] The prediction made by Raeder [2006] and supported observationally by Korotova et al. [2008] is that when the geomagnetic dipole tilt is nonzero, FTE signatures should be observed preferentially in the winter hemisphere. Raeder [2006] also predicts that FTE signatures should not be observed when the dipole tilt is near zero. We shall first discuss whether the observations fit this overall picture, and then discuss the impact of the other (nonseasonal) biases present in the data set.

[28] The orbit of the Cluster spacecraft crossed the postnoon magnetopause between November 2002 and February 2003, and the prenoon magnetopause between March and June 2003. Therefore, if the seasonal effect is present, we expect that the Cluster spacecraft should observe mainly standard polarity signatures (indicative of structures northward

Table 6. Summary Table for Figure $7^{\mathrm{a}}$

\begin{tabular}{lccccc}
\hline & & \multicolumn{2}{c}{ 'Positives' } & & 'Negatives' \\
\cline { 3 - 4 } \cline { 5 - 6 } & Prediction & $\begin{array}{c}\text { Standard } \\
\text { Signatures }\end{array}$ & $\begin{array}{c}\text { Reverse } \\
\text { Signatures }\end{array}$ & & $\begin{array}{c}\text { Crossings With } \\
\text { No Signatures }\end{array}$ \\
\hline Zone 1 & Standard & 60 & 0 & 3 \\
Zone 2 & None & 7 & 1 & 2 \\
Zone 3 & None & 2 & 0 & 4 \\
Zone 4 & None & 0 & 0 & 4 \\
Zone 5 & Reverse & 2 & 4 & \\
Zone 6 & None & 4 & 6 & 4 \\
Zone 7 & None & 0 & 3 & \\
Zone 8 & None & 9 & 0 & \\
Total & & 84 & 14 & \\
\hline
\end{tabular}

${ }^{\mathrm{a}}$ Same format as Table 2 . of the $\mathrm{X}$ line) postnoon and reverse polarity signatures (southward of the $\mathrm{X}$ line) prenoon.

[29] The influence of the seasonal effect is perhaps clearest when we consider positive IMF $\mathrm{B}_{Y}$ components (Figure 7 and discussed in section 3.3). If there were no seasonal bias and if FTEs are formed at a component reconnection line (or lines) which passes near the subsolar point and which tilts in response to the IMF $\mathrm{B}_{Y}$ component [Gonzalez and Mozer, 1974], then when $\mathrm{B}_{Y}>0$, standard polarity signatures should form northward and dawnward of a tilted reconnection line, and reverse polarity signatures should form southward and duskward of the reconnection line. Therefore, we might expect to observe most standard polarity signatures in the northern prenoon quadrant, and most reverse polarity signatures in the southern postnoon quadrant. The absence of clear reverse polarity signatures in the southern postnoon quadrant (Figure 7f) could in part be due to the higher latitude of the Southern Hemisphere magnetopause crossings compared with those in the north, but these crossings do take place in the Southern Hemisphere summer. Likewise, we would expect most of the standard polarity signatures to occur in the prenoon Northern Hemisphere for this IMF orientation, but the magnetopause crossings in this quadrant occur during the Northern Hemisphere summer. The main cluster of standard polarity signatures was observed in the Northern Hemisphere, but just duskward of the noon meridian (Figure 7e). The dipole tilts for these events were negative (i.e., Northern Hemisphere winter),

Table 7. Truth Table for Figure $7^{\mathrm{a}}$

\begin{tabular}{lcc}
\hline & $\begin{array}{c}\text { Positives } \\
\text { (Number of Signatures) }\end{array}$ & $\begin{array}{c}\text { Negatives } \\
\text { (Number of Crossings) }\end{array}$ \\
\hline True & 64 & 15 \\
False & 34 & 7 \\
Total & 98 & 22 \\
\hline
\end{tabular}

\footnotetext{
${ }^{\mathrm{a}} \mathrm{Same}$ format as Table 3
} 
and so these events are consistent with the dipole tilt bias predicted by Raeder [2006]. Most of the magnetopause crossings which occurred in the northern prenoon quadrant (during Northern Hemisphere summer) and in the southern postnoon quadrant (Southern Hemisphere summer) had no clear FTE signatures (Figure 7b).

[30] Considering the strongly southward IMF case (which is the closest case to that modeled by Raeder [2006]), in the absence of a seasonal effect we would expect a uniform distribution of standard polarity signatures in the Northern Hemisphere, and a uniform distribution of reverse polarity signatures in the south (perhaps with the exception of near $\mathrm{Y}_{G S M} \approx 0$, as these crossings occur mainly through or poleward of the cusp). Once the magnetopause crossings and FTE signatures which occurred when the dipole tilt was less than $6^{\circ}$ were removed (Figure 4 and section 3.1), we find that most of the standard polarity signatures occur in the postnoon Northern Hemisphere (Figure 4e), where the magnetopause crossings occur during Northern Hemisphere winter. Most of the reverse polarity signatures occur in the prenoon Southern Hemisphere (Figure 4f), during the Southern Hemisphere winter. The magnetopause crossings with no clear FTE signatures (Figure 4b) occur mainly in the prenoon Northern Hemisphere quadrant (i.e., during Northern Hemisphere summer) and in the postnoon Southern Hemisphere quadrant (Southern Hemisphere summer). The fact that there are differences between the data plotted in Figures $3 \mathrm{c}$ and $3 \mathrm{e}$ and Figures $4 \mathrm{c}$ and $4 \mathrm{e}$ indicates that FTEs are formed when the dipole tilt is small and the IMF is mainly southward. This appears to contradict the Raeder [2006] prediction that FTEs should not form when the dipole tilt is small. However, as discussed in section 3.1, the FTEs which were observed near equinox occurred when the IMF had a significant $\mathrm{B}_{X}$ component, whereas Raeder [2006] only investigated reconnection when $B_{X}$ was zero. Furthermore, since no simulations were reported for intermediate dipole tilt angles, it is not clear whether the 'threshold' tilt angle at which FTE signatures would start to appear should be large or small. The presence of four standard polarity FTE signatures in the northern prenoon quadrant in Figures 4c and 4e also appears to be inconsistent with the Raeder [2006] prediction. For two of the four events, the IMF tilt angle was close to $160^{\circ}$ and therefore only $20^{\circ}$ from the tilt angle used by Raeder [2006]. This shows that the predictions made by Raeder [2006] are not observed exactly. This is not entirely surprising, as all simulations are likely to provide a view of nature that is simplified to some extent. However, it does appear that the simulation predictions occur frequently enough to ensure that there is a tendency toward FTE signatures being observed in the winter hemisphere in both the present study and in the results reported by Korotova et al. [2008].

[31] The $215^{\circ}<\theta_{B}<290^{\circ}$ clock angle bin contains the most FTEs (Table 1), and we argue that this is for two reasons. First, as shown in Figure 1, when the IMF had a southward component the $\mathrm{B}_{Y}$ component was more likely to be negative than positive. Consequently, if the chance of observing an FTE is the same when there is a positive IMF $\mathrm{B}_{Y}$ component as when there is an equal but opposite $\mathrm{B}_{Y}$ component, then we would expect more signatures in this clock angle range as the clock angle criterion is satisfied more often. Secondly, we would argue that the orbital bias (whereby the postnoon magnetopause is observed when the dipole tilt is negative and the prenoon magnetopause is observed when the tilt is positive) combines with the seasonal effect predicted by Raeder [2006] to make this the most favorable IMF orientation for Cluster to observe FTEs. A subsolar component reconnection line that is tilted by a negative $\mathrm{B}_{Y}$ component would give rise to standard polarity signatures in the postnoon Northern Hemisphere, and reverse polarity signatures in the prenoon Southern Hemisphere. The magnetopause crossings in the postnoon Northern Hemisphere occurred in the Northern Hemisphere winter (between November 2002 and March 2003), and the magnetopause crossings in the prenoon Southern Hemisphere occurred during the Southern Hemisphere winter (March to June 2003), making FTE observations more likely if there is a bias toward the winter hemisphere.

[32] As we noted in section 3, the data set is subject to two other causes of bias which must be considered in addition to the interplay between the local time of the magnetopause crossings and any seasonal effect. First, the IMF distribution in Figure 1 is not uniform. Secondly the latitudes of the magnetopause crossings are higher in the Southern Hemisphere than in the north, which can lead to a larger number of Southern Hemisphere magnetopause crossings without clear FTE signatures. We have accounted for the nonuniform IMF distribution by examining the number of magnetopause crossings in each IMF bin which do not have clear FTE signatures. In order to reduce the effect of the different latitudes of Northern and Southern Hemisphere magnetopause crossings and the influence of the cusps, we shall focus on the Northern Hemisphere crossings (which are less likely to be affected by the cusp). The low proportion of clear FTE signatures in the prenoon northern quadrant (excluding nearequinox events) for all of the IMF conditions considered (Figures $4 \mathrm{c}$ and $4 \mathrm{e}, 6 \mathrm{c}$ and $6 \mathrm{e}$, and $7 \mathrm{c}$ and $7 \mathrm{e}$ ) cannot be explained by the nonuniform IMF distribution or the latitudes of the magnetopause crossings, since there are Northern Hemisphere magnetopause crossings (equatorward of the cusps) on which these IMF criteria are satisfied, but on which no clear FTE signatures are observed (Figures 4b, 6b, and $7 \mathrm{~b})$. A directly related observation which cannot be explained by the IMF distribution or latitude of the orbits is the fact that when the IMF is strongly duskward, the distribution of standard polarity signatures peaks strongly slightly duskward of $\theta_{L}=0$, rather than in the prenoon Northern Hemisphere quadrant (Figure 7e).

[33] The need to exclude near-equinox events is not consistent with a strict interpretation of the Raeder [2006] prediction, and there are a small number of signatures which are observed in the summer hemisphere (Figures $4 \mathrm{c}$ and $4 \mathrm{e}$ ). Therefore, we conclude that the effects predicted by Raeder [2006] are present to a sufficient degree to cause noticeable anisotropies in the distribution of FTE signatures, even if the seasonal bias is not a strict rule. Nonetheless, the presence of a seasonal effect appears to be more consistent with the Raeder [2006] simulation than with other simulations which do not find such a bias [Dorelli and Bhattacharjee, 2009; Tan et al., 2011], although none of the simulations match the observations exactly.

[34] A bias toward FTE signatures being observed in the winter hemisphere could, in principle, occur if FTEs are generated by single X line reconnection [Scholer, 1988; 


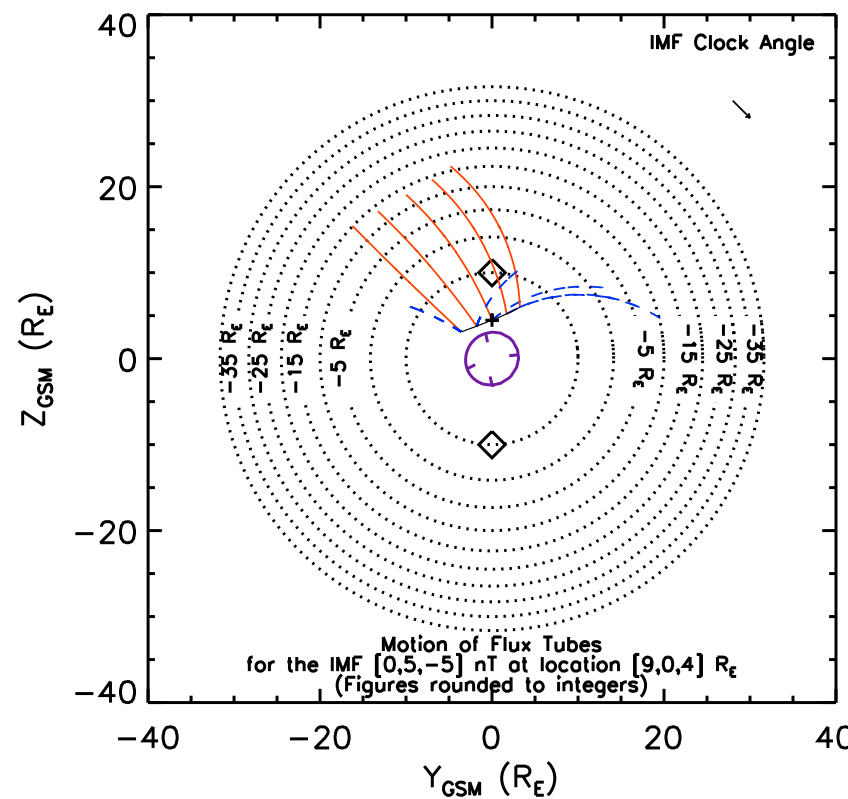

Figure 8. Results of the Cooling et al. [2001] model showing the expected paths of flux tubes connected to the Northern Hemisphere (red solid lines) and flux tubes connected to the Southern Hemisphere (blue dashed lines) if a reconnection line is initiated outside the sub-Alfvénic flow region at $(9.0,0,4.5)_{G S M} \mathrm{R}_{E}$. The model inputs were an interplanetary magnetic field of $(0,5,-5)_{G S M} \mathrm{nT}$, a solar wind density of $10 \mathrm{~cm}^{-3}$, and a solar wind speed of $450 \mathrm{~km} \mathrm{~s}^{-1}$. The boundary of the sub-Alfvénic flow region is marked by a purple circle; the location of the cusps is fixed at $\mathrm{X}_{G S M}=5 \mathrm{R}_{E}$ and $\mathrm{Z}_{G S M}= \pm 10 \mathrm{R}_{E}$. Concentric circles indicate contours of the magnetopause surface in $\mathrm{X}_{G S M}$.

Southwood et al., 1988] if for some reason the X line forms preferentially in the winter hemisphere and in the region where the magnetosheath flow is super Alfvénic. Figure 8 shows the results of a run of the Cooling et al. [2001] model of reconnected magnetic field line motion for a southward and duskward IMF, using typical solar wind conditions (a solar wind density of $10 \mathrm{~cm}^{-3}$ and a solar wind speed of $\left.450 \mathrm{~km} \mathrm{~s}^{-1}\right)$. The Cooling et al. [2001] model is a simple physics-based model which uses the Spreiter et al. [1966] model of magnetosheath plasma density and velocity and a magnetosheath magnetic field model developed by Kobel and Flückiger [1994] to calculate the expression derived by Cowley and Owen [1989] for the velocity of a reconnected magnetic field line at any point on the magnetopause. The results are shown projected into the Y-Z GSM plane, but the magnetopause surface is denoted by contours in $\mathrm{X}_{G S M}$. The region where the magnetosheath flow is below the local Alfvén speed is contained within the purple circle; outside this circle, the magnetosheath flow is super Alfvénic. A reconnection line has been initiated just outside this boundary, at $(9.0,0,4.5)_{G S M} \mathrm{R}_{E}$. The paths taken by reconnected magnetic field lines connected to the Northern Hemisphere are indicated by solid red lines, and the paths taken by field lines connected to the Southern Hemisphere are indicated by dashed blue lines. As the reconnection site is in the super-Alfvénic flow region, the pressure gradient force exerted by the magnetosheath plasma drags the field lines connected to the Southern Hemisphere tailward faster than the magnetic tension force can pull them equatorward [Cowley and Owen, 1989; Fear et al., 2005, 2007]. (Consequently, although reconnection may take place here, the reconnection line must move such that in its rest frame the plasma flow is sub-Alfvénic, and therefore reconnection cannot become a steady state process [Cowley and Owen, 1989].) As a result of the local super-Alfvénic magnetosheath flow, all of the reconnected field lines move northward, and so no FTE signatures would be observed at the magnetopause in the Southern Hemisphere. If, for some reason, the reconnection site regularly formed significantly northward of the magnetosheath stagnation point in the Northern Hemisphere winter, and southward of the stagnation point in the Southern Hemisphere winter, then the single $\mathrm{X}$ line reconnection process could give rise to a seasonal bias in the same sense as the Raeder [2006] model predicts, and was observed by Korotova et al. [2008] and in this study. Possible north-south asymmetries in the location of the reconnection line are still under investigation [Palmroth et al., 2012], but unless a mechanism is found that consistently moves a single $\mathrm{X}$ line toward the winter hemisphere then we suggest that the observed seasonal bias is more likely to be caused by sequential multiple $\mathrm{X}$ line reconnection as modeled by Raeder [2006].

[35] Finally in this section, we note that Raeder [2006] only carried out simulations for $\theta_{B}=165^{\circ}$ and $180^{\circ}$, and that most of the current observations are therefore outside the range of his simulations. We therefore conclude that the trends caused by the seasonal bias persist for a wider range of IMF orientations than modeled by Raeder [2006].

\subsection{Effective Tilt Dependence}

[36] In order to check the effect of the IMF tilt angle, we have also reordered the data by the effective dipole tilt, which combines the effects of the geomagnetic dipole tilt and the IMF tilt angle. We defined the IMF tilt as:

$$
\Phi_{I M F}=\arctan \left[B_{X} / B_{Z}\right] .
$$

This definition is chosen such that the IMF tilt has a range between $\pm 90^{\circ}$, and is unchanged if the IMF vector is rotated by $180^{\circ}$ in the X-Z GSM plane (i.e., there is no distinction in our definition between the tilt angle for the GSM IMF vectors $(+5,0,-1) \mathrm{nT}$ and $(-5,0,+1) \mathrm{nT})$. The effective tilt angle is then defined as:

$$
\Phi_{\text {eff }}=\Phi_{\text {dipole }}-\Phi_{I M F} .
$$

[37] Figure 9 shows the locations of the standard and reverse polarity FTE signatures sorted according to the IMF clock angle and the effective tilt. Each row contains data from one clock angle bin (indicated on the left), and the remaining columns show the location of standard and reverse polarity FTE signatures for positive and negative effective tilt angles. FTE signatures which occurred when the magnitude of the effective tilt angle was less than $10^{\circ}$ have been discarded. The effective tilt angle does not order the data well. This is seen most clearly in the standard polarity plots in the middle and bottom rows, (representing significant negative and positive IMF $\mathrm{B}_{Y}$ components). 

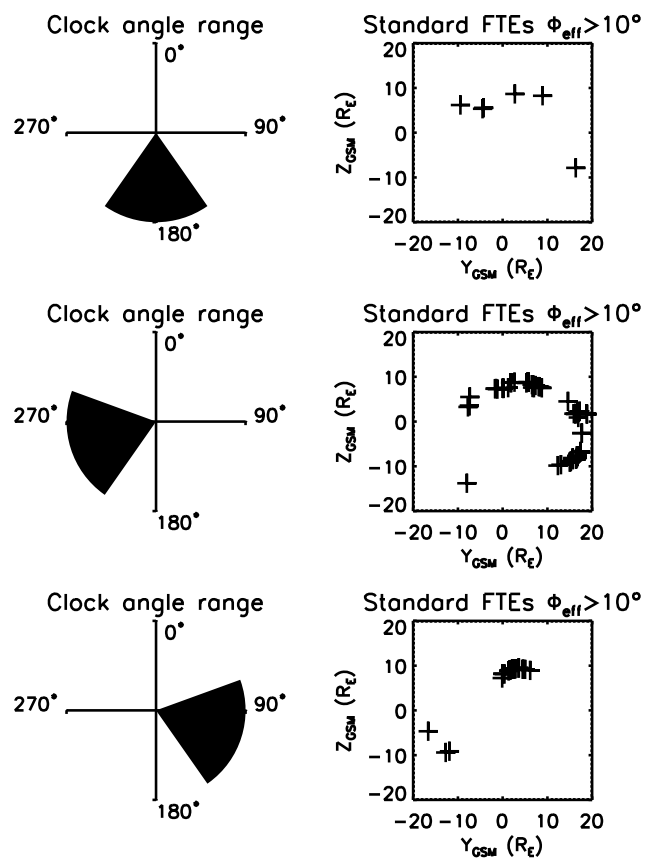

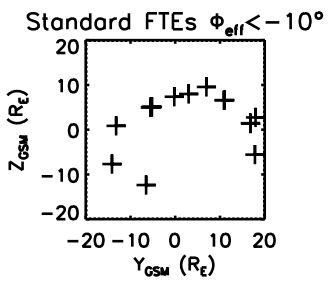

Standard FTEs $\Phi_{\text {eff }}<-10^{\circ}$
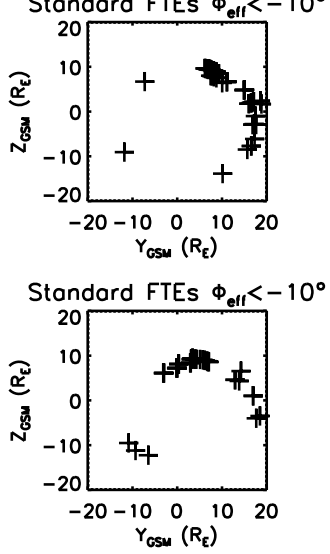
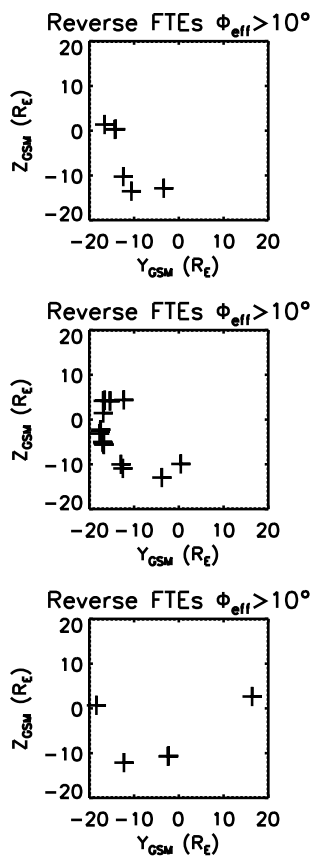
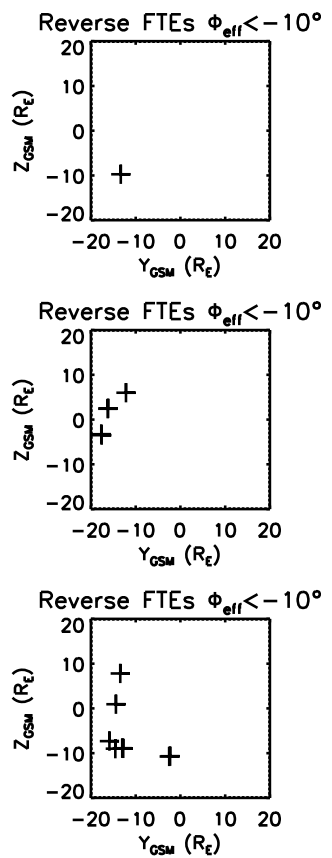

Figure 9. FTE data sorted by IMF clock angle and effective dipole tilt $\left(\Phi_{\text {eff }}\right)$. Each row contains data from one clock angle bin listed in Table 1 and indicated by the corresponding clock angle range shown. Shown are the locations of (from left) standard polarity FTE signatures when $\Phi_{\text {eff }}>10^{\circ}$, standard polarity signatures when $\Phi_{\text {eff }}<-10^{\circ}$, reverse polarity signatures when $\Phi_{\text {eff }}>10^{\circ}$, and reverse polarity signatures when $\Phi_{\text {eff }}<-10^{\circ}$.

Standard polarity signatures are observed plentifully in the postnoon Northern Hemisphere irrespective of whether the effective tilt angle is positive or negative. No other trend is evident.

[38] Lavraud et al. [2005] investigated the effect of effective dipole tilt on high-latitude reconnection for northward IMF conditions, and found the effect of IMF tilt angle was marginal compared with geomagnetic dipole tilt angle. They suggested that the potential effect of the IMF tilt may be partially removed by passage of the IMF through the bow shock. It also appears to be the case for the FTE signatures in this study that the effect of the IMF tilt angle is minimal compared with the effect of the geomagnetic dipole tilt.

\subsection{IMF Control of Peak FTE Location}

[39] The relationship between the locations of the FTE signatures and the IMF clock angle is further explored in Figure 10, which summarizes the locations of the standard polarity signatures in Figures 3-7 but in smaller IMF clock angle bins, and demonstrates that the IMF clock angle control of FTE location is evident with relatively small variations in clock angle. (Reverse polarity signatures are omitted, as there are fewer events and so further subdivision produces very small sample sizes, and as noted above they are less likely to be observed as Southern Hemisphere magnetopause crossings are more likely to occur through or poleward of the cusp. Standard polarity FTE signatures which occurred when the magnitude of the dipole tilt angle was less than $6^{\circ}$ have also been removed in the same manner as in Figure 4.) Figure 10 is split into two halves, representing negative $\mathrm{B}_{Y}$ (left) and positive $\mathrm{B}_{Y}$ (right). Within each half, each row contains three panels which show the IMF clock angle range pertaining to the panel, the locations of the standard polarity FTE signatures (again color coded by dipole tilt angle), and a histogram summarizing the location data. The IMF clock angle $\theta_{B}$ rotates anticlockwise as the panels progress down the left and side and then up the right hand side, such that the two halves of each row represent mirror image clock angles. When $270^{\circ}<\theta_{B}<290^{\circ}$ (Figure 10a), the mean location of the standard polarity FTE signatures is close to the equator and on the dusk side. As $\theta_{B}$ rotates anticlockwise to southward and dawnward orientations (Figures 10b and 10c) the mean location also rotates anticlockwise. The difference in the mean locations in Figures $10 \mathrm{~b}$ and $10 \mathrm{c}$ is small $\left(\sim 4^{\circ}\right)$, but the mean location is directed slightly more northward when $210^{\circ}<\theta_{B}<240^{\circ}$ (Figure 10c) than when $240^{\circ}<\theta_{B}<270^{\circ}$ (Figure 10b). The mean location does not change much when the IMF rotates further anticlockwise to $180^{\circ}<\theta_{B}<210^{\circ}$ (Figure 10d) and there is no clear mean direction when $150^{\circ}<\theta_{B}<180^{\circ}$ (Figure 10e). Once the IMF rotates further anticlockwise to orientations with a positive $\mathrm{B}_{Y}$ component that is comparable to or greater than $\left|B_{Z}\right|$ (Figures 10f, 10g, and 10h), the anticlockwise rotation continues from the orientation in

Figure 10. (a-e) The standard polarity events represented in Figures 3, 6, and 7, rebinned into smaller IMF clock angle bins. Magnetopause crossings and FTE signatures with a dipole tilt magnitude less than $6^{\circ}$ have been removed. The color coding represents the geomagnetic dipole tilt, indicated by the same color scale in Figure 3, which is omitted for reasons of space. 

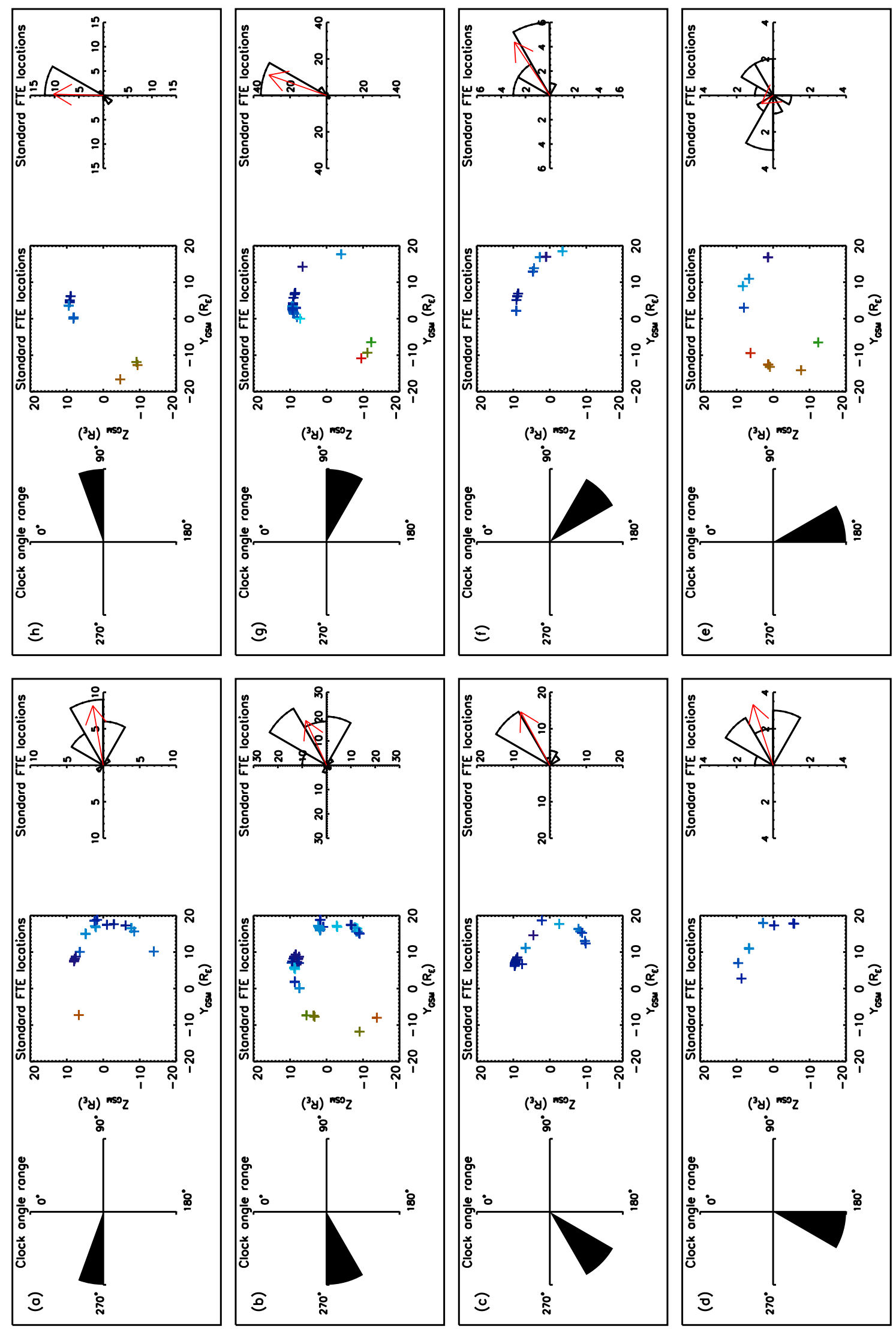

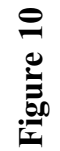


Figure 10c until the mean location vector is directed northward (Figure 10h).

[40] In summary, as the IMF rotates from a slightly northward orientation that is dominated by a negative $\mathrm{B}_{Y}$ component (Figure 10a), via southward to a slightly northward orientation dominated by a positive $\mathrm{B}_{Y}$ component (Figure $10 \mathrm{~h}$ ), the mean location rotates through about $90^{\circ}$. If data from the prenoon magnetopause were also available from November 2002 to February 2003, and data from the postnoon magnetopause were available from February to June 2003 (thus eliminating the combined orbital-seasonal bias), then we would expect to see a $\sim 180^{\circ}$ rotation of the mean FTE location vector. The fact that the distribution of standard polarity events undergoes a $90^{\circ}$ rotation in Figure 10 from peaking near $\theta_{L}=90^{\circ}$ when $\theta_{B} \approx 270^{\circ}$ to a peak near $\theta_{L}=0^{\circ}$ when $\theta_{B} \approx 90^{\circ}$, rather than a symmetric rotation about $\theta_{L}=0^{\circ}$ as might be expected by solely considering a tilted reconnection line, is consistent with the prediction that FTE signatures are less likely to be observed in the winter hemisphere. Since we consider only standard polarity signatures and consider $\theta_{B}$ bins separately, this observation cannot be explained the different latitudes of Northern and Southern Hemisphere magnetopause crossings, or by the nonuniform $\theta_{B}$ distribution.

[41] The presence of a preference for FTE signatures being observed in the winter hemisphere is not inconsistent with earlier statistics reported by Berchem and Russell [1984], who used data from 5 years of ISEE magnetopause crossings (October to December 1977, July to December 1978, June 1979 to January 1980, June 1980 to January 1981, and June 1981 to January 1982). This resulted in reasonably uniform coverage of the dayside magnetopause between $-5 \mathrm{R}_{E}<$ $\mathrm{Z}_{G S E}<+5 \mathrm{R}_{E}$ and $-15 \mathrm{R}_{E}<\mathrm{Y}_{G S E}<+15 \mathrm{R}_{E}$ [Berchem and Russell, 1984, Figure 1]. The postnoon magnetopause was sampled between June and October, and the prenoon magnetopause was observed between October and January. When the locations of the ISEE magnetopause crossings are plotted in GSM coordinates, the dipole tilt introduces an orbital bias toward more southerly latitudes at the postnoon magnetopause (mostly observed between the Southern Hemisphere winter solstice and equinox) and to more northerly latitudes prenoon (observed after the equinox and during the Northern Hemisphere winter). Therefore, throughout each magnetopause crossing season, the orbits of the ISEE spacecraft were biased toward the winter hemisphere.

\section{Conclusions}

[42] We have presented an analysis of the seasonal dependence of previously reported FTE signatures observed by Cluster. Although several FTE signatures were observed in locations that are not consistent with the seasonal bias predicted by Raeder [2006], we find that the seasonal bias is present and strong enough that the statistical distributions are affected by it. We therefore confirm the INTERBALL observations [Korotova et al., 2008] that in situ FTE signatures are more likely to be observed in the winter hemisphere, as predicted by Raeder [2006]. The combined effect of the IMF tilt angle and the geomagnetic dipole tilt angle appears to be much less significant than the effect of the dipole tilt alone. It is possible that FTE signatures caused by single $\mathrm{X}$ line reconnection could have such a seasonal bias if the $\mathrm{X}$ line was regularly shifted away from the magnetosheath stagnation point into the winter hemisphere and into the region of super-Alfvénic magnetosheath flow. However, in the absence of an explanation of why a single $X$ line should regularly be shifted in such a manner, we believe that our observations are more easily understood in the context of sequential multiple $\mathrm{X}$ line reconnection as modeled by Raeder [2006]. We find that if FTE signatures observed away from the subsolar region are examined, and the seasonal bias predicted by Raeder [2006] is taken into account, the location where the largest number of standard polarity FTE signatures is observed clearly rotates in the GSM Y-Z plane as $\mathrm{B}_{Y}$ changes.

[43] Acknowledgments. R.C.F. would like to acknowledge discussions with members of the International Space Science Institute international team on "Advances in understanding of the structure and dynamics of flux transfer at the Earth's magnetopause" (A. Marchaudon, K. A. McWilliams, C. J. Owen, J. Raeder, D. G. Sibeck, Y. Wang, and J. A. Wild), and would like to thank D. J. Andrews for suggesting the application of directional statistics. Work at the University of Leicester was supported by STFC grant ST/H002480/1. We also thank the referees for their constructive comments.

[44] Masaki Fujimoto thanks the reviewers for their assistance in evaluating this paper.

\section{References}

Balogh, A., et al. (2001), The Cluster Magnetic Field Investigation: Overview of in-flight performance and initial results, Ann. Geophys., 19, 1207-1217, doi:10.5194/angeo-19-1207-2001.

Berchem, J., and C. T. Russell (1984), Flux transfer events on the magnetopause: Spatial distribution and controlling factors, J. Geophys. Res., 89 (A8), 6689-6703, doi:10.1029/JA089iA08p06689.

Cooling, B. M. A., C. J. Owen, and S. J. Schwartz (2001), Role of the magnetosheath flow in determining the motion of open flux tubes, $J$. Geophys. Res., 106(A9), 18,763-18,775, doi:10.1029/2000JA000455.

Cowley, S. W. H., and C. J. Owen (1989), A simple illustrative model of open flux tube motion over the dayside magnetopause, Planet. Space Sci., 37, 1461-1475, doi:10.1016/0032-0633(89)90116-5.

Daly, P. W., M. A. Saunders, R. P. Rijnbeek, N. Sckopke, and C. T. Russell (1984), The distribution of reconnection geometry in flux transfer events using energetic ion, plasma and magnetic data, J. Geophys. Res., 89(A6), 3843-3854, doi:10.1029/JA089iA06p03843.

Dorelli, J. C., and A. Bhattacharjee (2009), On the generation and topology of flux transfer events, J. Geophys. Res., 114, A06213, doi:10.1029/ 2008JA013410.

Fear, R. C., A. N. Fazakerley, C. J. Owen, and E. A. Lucek (2005), A survey of flux transfer events observed by Cluster during strongly northward IMF, Geophys. Res. Lett., 32, L18105, doi:10.1029/2005GL023811.

Fear, R. C., S. E. Milan, A. N. Fazakerley, C. J. Owen, T. Asikainen, M. G. G. T. Taylor, E. A. Lucek, H. Rème, I. Dandouras, and P. W. Daly (2007), Motion of flux transfer events: A test of the Cooling model, Ann. Geophys., 25, 1669-1690, doi:10.5194/angeo-25-1669-2007.

Fear, R. C., S. E. Milan, J. Raeder, and D. G. Sibeck (2010), Asymmetry in the bipolar signatures of flux transfer events, J. Geophys. Res., 115, A11217, doi:10.1029/2010JA015363.

Gonzalez, W. D., and F. S. Mozer (1974), A quantitative model for the potential resulting from reconnection with an arbitrary interplanetary magnetic field, J. Geophys. Res., 79(28), 4186-4194, doi:10.1029/ JA079i028p04186.

Kobel, E., and E. O. Flückiger (1994), A model of the steady state magnetic field in the magnetosheath, J. Geophys. Res., 99(A12), 23,617-23,622, doi:10.1029/94JA01778.

Korotova, G. I., D. G. Sibeck, and T. Rosenberg (2008), Seasonal dependence of Interball flux transfer events, Geophys. Res. Lett., 35, L05106, doi:10.1029/2008GL033254

Lavraud, B., M. F. Thomsen, M. G. G. T. Taylor, Y. L. Wang, T. D. Phan, S. J. Schwartz, R. C. Elphic, A. Fazakerley, H. Rème, and A. Balogh (2005), Characteristics of the magnetosheath electron boundary layer under northward interplanetary magnetic field: Implications for highlatitude reconnection, J. Geophys. Res., 110, A06209, doi:10.1029/ 2004JA010808. 
Lee, L. C., and Z. F. Fu (1985), A theory of magnetic flux transfer at the Earth's magnetopause, Geophys. Res. Lett., 12(2), 105-108, doi:10.1029/GL012i002p00105.

Mardia, K. V., and P. E. Jupp (2000), Directional Statistics, John Wiley, Chichester, U. K.

McComas, D. J., S. J. Bame, P. Barker, W. C. Feldman, J. L. Phillips, P. Riley, and J. W. Griffee (1998), Solar Wind Electron Proton Alpha Monitor (SWEPAM) for the Advanced Composition Explorer, Space Sci. Rev., 86(1-4), 563-612, doi:10.1023/A:1005040232597.

Milan, S. E., M. Lester, S. W. H. Cowley, and M. Brittnacher (2000), Convection and auroral response to a southward turning of the IMF: Polar UVI, CUTLASS, and IMAGE signatures of transient magnetic flux transfer at the magnetopause, J. Geophys. Res., 105(A7), 15,741-15,755, doi:10.1029/2000JA900022.

Omidi, N., and D. G. Sibeck (2007), Flux transfer events in the cusp, Geophys. Res. Lett., 34, L04106, doi:10.1029/2006GL028698.

Palmroth, M., R. C. Fear, and I. Honkonen (2012), Magnetopause energy transfer dependence on the interplanetary magnetic field and the Earth's magnetic dipole axis orientation, Ann. Geophys., in press.

Paschmann, G., G. Haerendel, I. Papamastorakis, N. Sckopke, S. J. Bame, J. T. Gosling, and C. T. Russell (1982), Plasma and magnetic field characteristics of magnetic flux transfer events, J. Geophys. Res., 87(A4), 2159-2168, doi:10.1029/JA087iA04p02159.

Phan, T. D., J. T. Gosling, G. Paschmann, C. Pasma, J. F. Drake, M. Øieroset, D. Larson, R. P. Lin, and M. S. Davis (2010), The dependence of magnetic reconnection on plasma $\beta$ and magnetic shear: Evidence from solar wind observations, Astrophys. J., 719, L199-L203, doi:10.1088/2041-8205/719/2/L199.

Pinnock, M., A. S. Rodger, J. R. Dudeney, K. B. Baker, P. T. Newell, R. A. Greenwald, and M. E. Greenspan (1993), Observations of an enhanced convection channel in the cusp ionosphere, J. Geophys. Res., 98(A3), 3767-3776, doi:10.1029/92JA01382.

Provan, G., T. K. Yeoman, and S. E. Milan (1998), CUTLASS Finland radar observations of the ionospheric signatures of flux transfer events and the resulting plasma flows, Ann. Geophys., 16, 1411-1422, doi:10.1007/s00585-998-1411-0.

Raeder, J. (2006), Flux transfer events: 1. Generation mechanism for strong southward IMF, Ann. Geophys., 24(1), 381-392, doi:10.5194/angeo-24381-2006.

Rijnbeek, R. P., S. W. H. Cowley, D. J. Southwood, and C. T. Russell (1984), A survey of dayside transfer events observed by ISEE 1 and
2 magnetometers, J. Geophys. Res., 89(A2), 786-800, doi:10.1029/ JA089iA02p00786.

Russell, C. T., and R. C. Elphic (1978), Initial ISEE magnetometer results: Magnetopause observations, Space Sci. Rev., 22(6), 681-715, doi:10.1007/BF00212619.

Russell, C. T., J. Berchem, and J. G. Luhmann (1985), On the source region of flux transfer events, Adv. Space Res., 5, 363-368, doi:10.1016/02731177(85)90162-0.

Sandholt, P. E., C. S. Deehr, A. Egeland, B. Lybekk, R. Viereck, and G. J. Romick (1986), Signatures in the dayside aurora of plasma transfer from the magnetosheath, J. Geophys. Res., 91(A9), 10,063-10,079, doi:10.1029/JA091iA09p10063.

Scholer, M. (1988), Magnetic flux transfer at the magnetopause based on single X line bursty reconnection, Geophys. Res. Lett., 15(4), 291-294, doi:10.1029/GL015i004p00291.

Smith, C. W., J. L'Heureux, N. F. Ness, M. H. Acuña, L. F. Burlaga, and J. Scheifele (1998), The ACE Magnetic Fields Experiment, Space Sci. Rev., 86(1-4), 613-632, doi:10.1023/A:1005092216668.

Southwood, D. J., C. J. Farrugia, and M. A. Saunders (1988), What are flux transfer events?, Planet. Space Sci., 36(5), 503-508, doi:10.1016/00320633(88)90109-2.

Spreiter, J. R., A. L. Summers, and A. Y. Alksne (1966), Hydromagnetic flow around the magnetosphere, Planet. Space Sci., 14, 223-253, doi:10.1016/0032-0633(66)90124-3.

Tan, B., Y. Lin, J. D. Perez, and X. Y. Wang (2011), Global-scale hybrid simulation of dayside magnetic reconnection under southward IMF Structure and evolution of reconnection, J. Geophys. Res., 116 , A02206, doi:10.1029/2010JA015580.

Wild, J. A., et al. (2001), First simultaneous observations of flux transfer events at the high-latitude magnetopause by the Cluster spacecraft and pulsed radar signatures in the conjugate ionosphere by the CUTLASS and EISCAT radars, Ann. Geophys., 19, 1491-1508, doi:10.5194/ angeo-19-1491-2001.

R. C. Fear and S. E. Milan, Department of Physics and Astronomy, University of Leicester, Leicester LE1 7RH, UK. (r.fear@ion.le.ac.uk)

M. Palmroth, Finnish Meteorological Institute, PO Box 503, FI-00101 Helsinki, Finland. 